\title{
Chapter
}

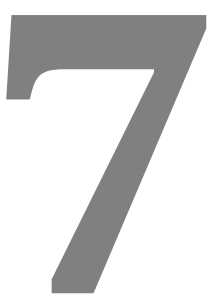

\section{THE IMPORTANCE OF CHOOSING THE OPTIMAL SOLID FORM OF A DRUG}

\section{Rolf Hilfiker}

\section{Contents}

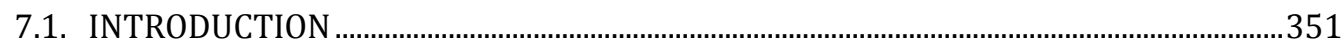

7.2. THERMODYNAMICS.......................................................................................................354

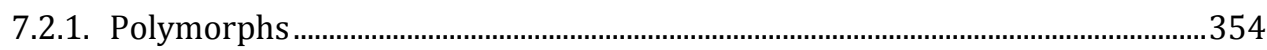

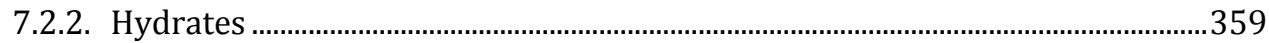

7.2.2.1. Example for the Selection of a Hydrate or Anhydrate..........................362

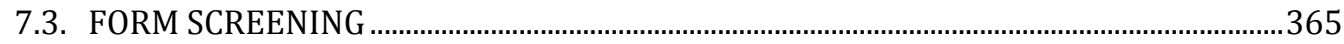

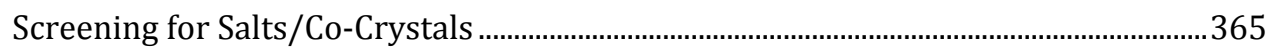

7.3.1.1 Example of a Co-Crystal Screen …………………………………………....366

7.3.2. Screening for Polymorphs .....................................................................................368

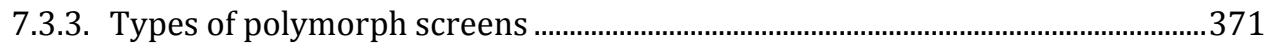

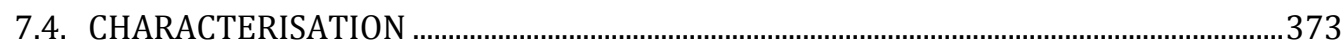

7.4.1. X-ray diffraction (XRD) ..........................................................................................

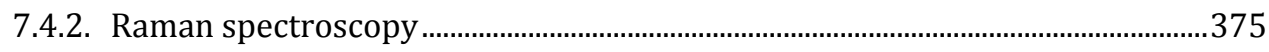




\section{Chapter 7}

7.5. CONCLUSION 377

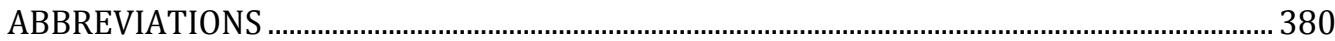

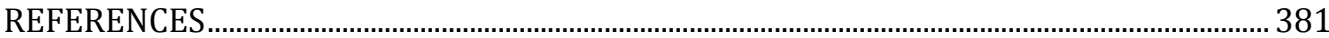




\subsection{INTRODUCTION}

The majority of drugs are administered as oral dosage forms, and by far the most common oral dosage forms are tablets and capsules. This means that in the formulated product (drug product, DP), the active pharmaceutical ingredient (API) or drug substance (DS) will be in most cases in the solid state. This fact presents at the same time big challenges and big opportunities to drug developers, since a particular active molecule can in most cases exist in many different solid forms with very different physical properties.

First of all, the active molecule itself can often be crystallised as different polymorphs. According to McCrone's definition [1], "The polymorphism of any element or compound is its ability to crystallise as more than one distinct crystal species," we will call different crystal arrangements of the same chemical composition polymorphs. Due to the different spatial arrangement of the molecules in the crystal lattice, different inter- and intra-molecular interactions such as van der Waals interactions and hydrogen bonds will be encountered in different crystal structures. Therefore, different polymorphs will have different free energies and consequently different physical properties such as solubility, chemical stability, melting point, density, etc. It is very important to note that while this definition is used most commonly, some authors, in particular the International Conference on Harmonisation (ICH), use the term "polymorph" more broadly, including both the amorphous state and solvates [2].

If long-range order is absent in the solid phase, the substance is called amorphous. According to the third law of thermodynamics, the stable form at $0 \mathrm{~K}$ should always be a perfectly ordered one. Therefore, amorphous forms are metastable, i.e. have a higher free energy and a higher solubility than the stable crystalline phase. Generally, they are also chemically much less stable than a crystalline form. Due to their higher solubility, they may be an attractive choice to solve solubility issues of poorly soluble drugs. But, the challenge is then to find ways to ensure that the amorphous form is sufficiently kinetically stable, so that crystallisation does not occur during storage.

Many compounds can also form solvates, where solvent molecules are incorporated in the crystal lattice. Different types of solvates exist and they can be stoichiometric or non-stoichiometric. In stoichiometric solvates, the solvent molecule is usually an integral part of the crystal structure and is essential for the maintenance of the molecular network (see Figure 7.1). Non-stoichiometric 
solvates can, for example, be channel solvates, where the solvent molecules reside in channels in the crystal structure. Of particular practical importance are hydrates, where the incorporated solvent is water. Solvates, with the exception of hydrates, are normally not a preferred form for the API. The first reason is that unnecessary addition of solvents to the drug product is undesirable; the second, and probably more important one, is that solvates are never thermodynamically stable at ambient conditions, since the vapour pressure of any solvent in the atmosphere is zero, which means that under equilibrium conditions, the solvate will desolvate, i.e. the solvent molecules will leave the crystal structure.

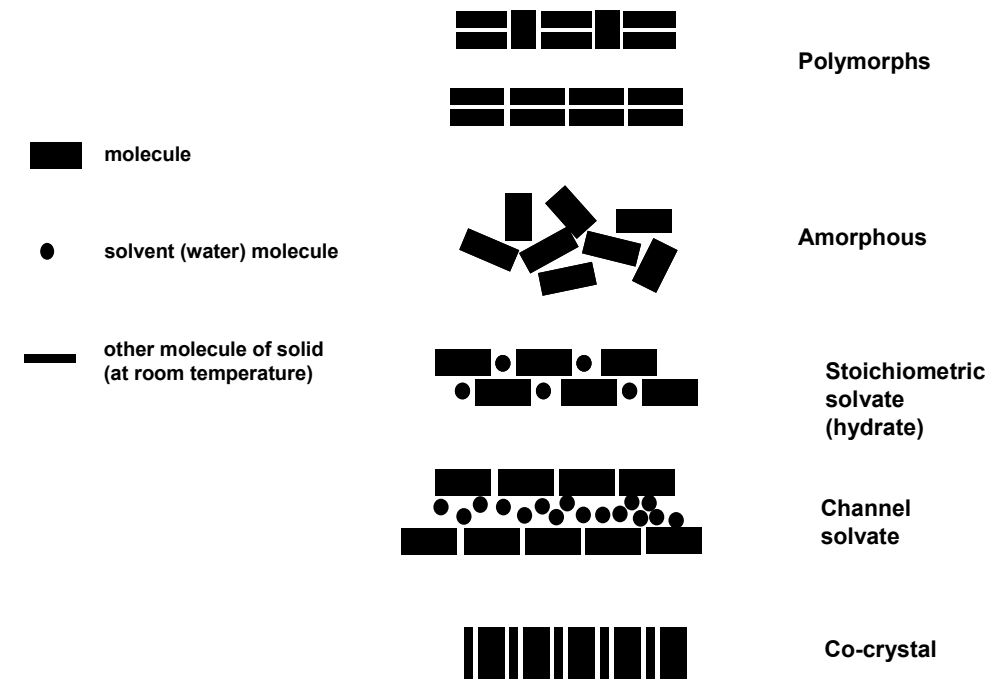

Figure 7.1. Schematic depiction of various types of solid forms

This situation is different for co-crystals. In co-crystals, the additional molecule which is incorporated in the crystal lattice is a solid at room temperature. Therefore, its equilibrium vapour pressure is negligible for practical purposes, and a co-crystal can be thermodynamically stable at ambient conditions, which can make co-crystals very viable candidates as drug substances.

When a compound is acidic or basic, it is generally possible to create a salt with a suitable base or acid, and such a salt can in turn often be crystallised. Since salts usually have higher water solubility and bioavailability than the corresponding uncharged molecule, they are popular choices for drug substances. About half of all active molecules are marketed as salts $[3,4]$.

Obviously, salts may also crystallise as solvates or co-crystals; solvates, cocrystals and salts, together with their solvates and co-crystals, may all be able to exist as different polymorphs. This means that the potential number of solid forms of an API is indeed very large, which implies on one hand that significant effort is needed to produce and characterise all relevant forms, but on the other hand, it also means that there is a huge potential to optimise the properties of the solid according to the specific requirement of the drug. Of fundamental importan- 
ce to the decision as to which form to develop is the understanding of the thermodynamic relationship between the forms.

Therefore, the following steps should typically be followed in terms of solid-state investigations during the development of a new drug:

- $\quad$ screening for salts and/or co-crystals if the properties of the parent molecule are not optimal

- screening for polymorphs, hydrates and solvates of the parent molecule, salt or co-crystal

- characterisation of the various forms and elucidating their thermodynamic and kinetic relationships

- $\quad$ selection of the optimal form

- formulation development

- development of analytical methods to assess form purity

- development of a stable and efficient crystallisation process to produce the desired form

Polymorphism is a very common phenomenon in connection with drug substances where the API is a small organic molecule with a molecular weight below $600 \mathrm{~g} / \mathrm{mol}[3,5]$. Literature values for the prevalence of polymorphs, solvates and hydrates vary. Figure 7.2a is the result of 180 focused polymorph screens carried out at a contract research organisation [6] and Figure 7.2b shows the statistical evaluation of entries in the European Pharmacopoeia with regard to solid-state information [7]. It is not surprising that the prevalence of different solid forms found in the European Pharmacopoeia is lower than for the recently carried out polymorph screens, since some of the API in the Pharmacopeia were discovered a long time ago, when polymorph screens were not always carried out thoroughly. The data show that the prevalence of substances which can exist in various solid forms is high and that if considerable effort is put into polymorph screening, several solid forms will be discovered for the vast majority (>85\%) of substances.

(a)

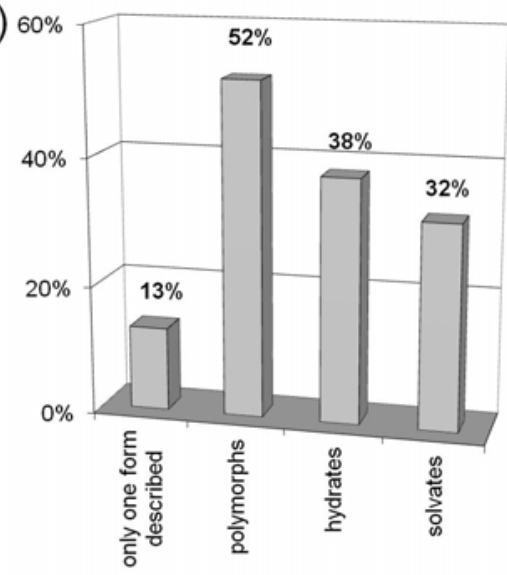

(b)

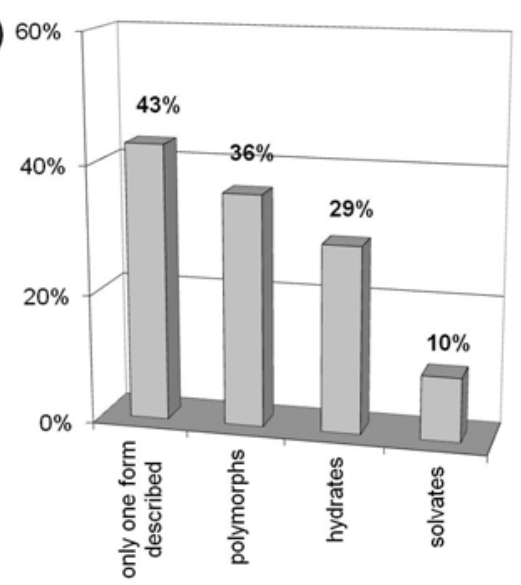

Figure 7.2. Frequency of occurrence of solid forms of organic molecules, (a) result of 180 focused polymorph screens; (b) from data in the European Pharmacopoeia 
The importance, implications and investigation of polymorphism is described indepth in several recent monographs and edited books [8-12]. An excellent book about salts in the pharmaceutical industry is the one by Stahl \& Wermuth [4].

Even if the envisaged market form of the drug is not a solid but a solution, information about the solid-state properties of the drug substance may still be necessary [13]. If different forms have significantly different solubilities, it may be possible to unintentionally create a supersaturated solution with respect to the least soluble form by preparing a concentrated solution of a metastable form. Furthermore, the drug substance will in most cases be handled as a solid in some stages of the manufacturing process, and its handling and stability properties may depend critically on the solid form.

In addition to the crystalline, amorphous and liquid states, condensed matter can exist in various mesophases. These mesophases are characterised by exhibiting partial order between that of the crystalline and amorphous states $[14,15]$. Several drug substances are known to form liquid crystalline phases, which can be either thermotropic, where liquid crystal formation is induced by temperature, or lyotropic, where the transition is solvent-induced [16-18]. Such mesophases as well as the amorphous form will not be further discussed in this chapter.

\subsection{THERMODYNAMICS}

Thermodynamics describes which form is stable under which condition as a function of external conditions (temperature, pressure, humidity, etc.). It is clearly essential to know which form is stable under given conditions, since any other form may convert to this stable form during storage. Such a transition will change the properties of the drug and may therefore affect its efficacy or safety. In the absence of humidity, there will be exactly one non-solvated polymorph of a particular compound (active molecule, salt or co-crystal) which is thermodynamically stable at a certain temperature and pressure (except at the transition temperature of an enantiotropic system; see below). Under ambient conditions, i.e. at a certain temperature and humidity, a hydrate may be the thermodynamically stable form. This is the reason why, in most cases, either a hydrate or the thermodynamically stable non-solvated form is developed. It ensures that no conversion takes place during storage. The disadvantage of the stable form is that it will have the lowest solubility and therefore also the lowest bioavailability if it is a BCS (Biopharmaceutical Classification System) class II or IV drug [19].

\subsubsection{Polymorphs}

At constant pressure, the stability of a compound or a mixture of compounds is determined by its Gibbs free energy $(G)$. The temperature dependence of the basic thermodynamic state functions enthalpy $(H)$, entropy $(S)$ and Gibbs free energy is given by Equations 7.1 to 7.3 , where $c_{\mathrm{P}, \mathrm{m}}$ is the molar heat capacity at 
constant pressure, $T$ is the absolute temperature, $H^{0}$ is the enthalpy at $0 \mathrm{~K}$ and $S^{0}$ is the entropy at $0 \mathrm{~K}$ [20]. According to the third law of thermodynamics, $S^{0}$ is zero for a perfect crystal (see Introduction).

$$
\begin{aligned}
& H=H^{0}+\int_{0}^{T} c_{\mathrm{P}, \mathrm{m}} \mathrm{d} T \\
& S=S^{0}+\int_{0}^{T} \frac{c_{\mathrm{P}, \mathrm{m}}}{T} \mathrm{~d} T \\
& G=H-T S
\end{aligned}
$$

The temperature dependence of $H, \mathrm{~S}, T S$ and $G$ of any solid is depicted schematically in Figure 7.3.

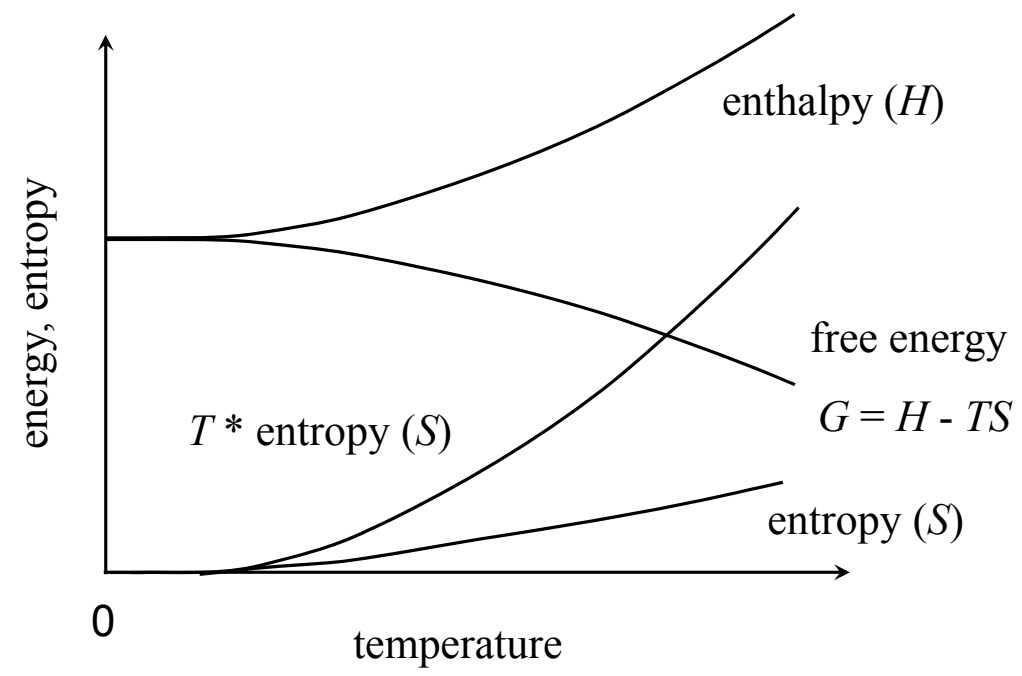

Figure 7.3. Temperature dependence of enthalpy $(H)$, free energy $(G)$, entropy $(S)$ and $T S$

Using these equations, we can now calculate the stability of polymorphs and the amorphous form (or melt) as a function of temperature. These could be either polymorphs of a single molecule, a particular solvate, salt or co-crystal. In Figure 7.4, the enthalpy and free energy of two polymorphs and the amorphous form/melt are plotted as a function of temperature. This is called an energytemperature diagram or ET diagram. The phase with the lowest free energy at any temperature is the thermodynamically stable one at that particular temperature. Thus, the melting point $\left(T_{\mathrm{m}}\right)$ of a polymorph is defined as the temperature where the free energy of that polymorph is equal to the free energy of the melt. If two different polymorphs ( $\alpha$ and $\beta$ ) exist, two different situations are now possible: 
(i) the free energy functions of polymorph $\alpha$ and polymorph $\beta$ do not intersect at a temperature below the melting point of polymorph $\beta$ (Figure 7.4a) and

(ii) the free energy functions of polymorph $\alpha$ and polymorph $\beta$ do intersect at a temperature which is lower than the melting point of polymorph $\beta$ (Figure 7.4b).

If (i) is the case, the system of polymorph $\alpha$ and $\beta$ is called a monotropic system, which means that polymorph $\alpha$ is more stable than polymorph $\beta$ at all temperatures below the melting point of the higher melting form. In situation (ii), the system of polymorph $\alpha$ and $\beta$ is called an enantiotropic system. In such a situation, polymorph $\beta$ is the thermodynamically stable form from $0 \mathrm{~K}$ to the transition temperature $\left(T_{\mathrm{t}}\right)$ and polymorph $\alpha$ is the stable form from the transition temperature to its melting point $\left(T_{\text {melt, } \alpha}\right)$. If more than two polymorphs exist, the above arguments can be extended in an analogous way.
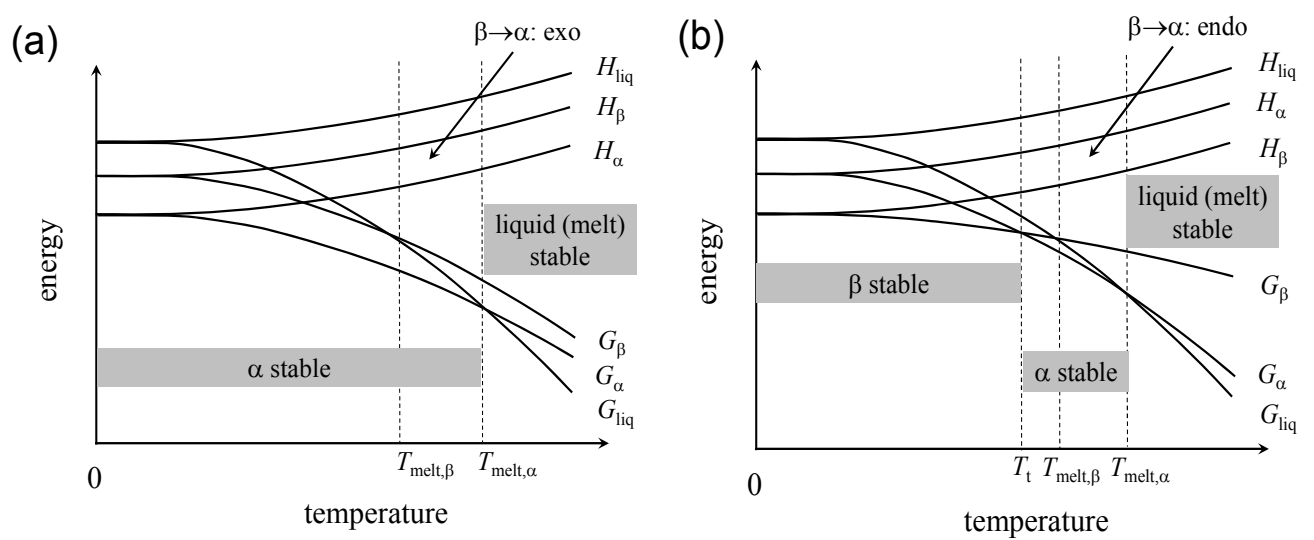

Figure 7.4. Qualitative energy-temperature (ET) diagram diagram for (a) a monotropic and (b) an enantiotropic system. The temperature regions of the stable forms are marked

It is very important to know if a system is monotropic or enantiotropic and what the transition temperature is in an enantiotropic system. If, for example, the transition temperature is $40^{\circ} \mathrm{C}$ and the low temperature form is chosen for development, it has to be ascertained that form conversion to the high temperature form will not occur during the processing steps or storage where higher temperatures may occur. It will also impact the design of the crystallisation process. If crystallisation starts at a temperature above the transition temperature, it must be ensured that the metastable form nucleates first, if the stable form at room temperature is the target and if a phase transformation during the crystallisation process is to be avoided, which is generally the case.

Constructing quantitative energy-temperature diagrams of the form in Figure 7.4 is experimentally difficult, since it requires knowledge of the enthalpies and heat capacities starting from $0 \mathrm{~K}$, a range which is very difficult to access. If, however, 
the enthalpy and free energy difference between the polymorph and amorphous form/melt is plotted as a function of temperature, quantitative energytemperature diagrams are readily accessible. Taking into account that the free energy difference between the melt and the polymorph at the melting point of the polymorph is zero, Equations 7.4 and 7.5 are obtained, which allow accurate calculation of enthalpy and free energy differences in the temperature range where the heat capacity can be determined. Naturally, the range close to $0 \mathrm{~K}$ will still be difficult to access, but it is normally of no practical interest anyway. From such a quantitative diagram, the transition temperature, the heat of transition between polymorphs (see Chapter 8) and the free energy difference and hence solubility ratio of polymorphs are obtained. $\Delta H_{\mathrm{m}, \alpha}=H_{\mathrm{liq}}-H_{\alpha}$ at $T_{\mathrm{m}, \alpha}$ is the melting enthalpy of polymorph $\alpha$.

$$
\begin{aligned}
& H_{\alpha}-H_{\mathrm{liq}}=-\Delta H_{\mathrm{m}, \alpha}+\int_{T_{\mathrm{m}}}^{T}\left(c_{\mathrm{P}, \mathrm{m}, \alpha}-c_{P, \mathrm{~m}, \mathrm{liq}}\right) \mathrm{d} T \\
& G_{\alpha}-G_{\mathrm{liq}}=-\Delta H_{\mathrm{m}, \alpha}+\int_{T_{\mathrm{m}}}^{T}\left(c_{\mathrm{P}, \mathrm{m}, \alpha}-c_{\mathrm{P}, \mathrm{m}, \mathrm{liq}}\right) \mathrm{d} T- \\
& -T\left(\frac{-\Delta H_{\mathrm{m}, \alpha}}{T_{\mathrm{m}, \alpha}}+\int_{T_{\mathrm{m}}}^{T} \frac{c_{\mathrm{P}, \mathrm{m}, \alpha}-c_{\mathrm{P}, \mathrm{m}, \mathrm{liq}}}{T} \mathrm{~d} T\right)
\end{aligned}
$$

If we make the further simplifying assumption that the heat capacity differences between solid and liquid are zero in the temperature range of interest, Equation 7.6 follows.

$$
G_{\alpha}-G_{\text {liq }} \approx-\left(\Delta H_{\mathrm{m}, \alpha} / T_{\mathrm{m}, \alpha}\right)\left(T_{\mathrm{m}, \alpha}-T\right)
$$

Therefore, using experimentally determined values of $T_{\mathrm{m}}$ and $\Delta H_{\mathrm{m}}$ (see Chapter 8), quantitative energy-temperature diagrams can be conveniently constructed with the simplifying assumption stated above (see Figure 7.5). The slopes of the lines are equal to the ratio of melting enthalpy and melting temperature of the corresponding polymorph.

From Equation 7.6, the transition temperature of enantiotropic systems can then also be easily calculated (Equation 7.7); see also Chapter 8.

$$
T_{\mathrm{t}}=\frac{T_{\mathrm{m}, \alpha} T_{\mathrm{m}, \beta}\left(\Delta H_{\mathrm{m}, \beta}-\Delta H_{\mathrm{m}, \alpha}\right)}{T_{\mathrm{m}, \alpha} \Delta H_{\mathrm{m}, \beta}-T_{\mathrm{m}, \beta} \Delta H_{\mathrm{m}, \alpha}}
$$



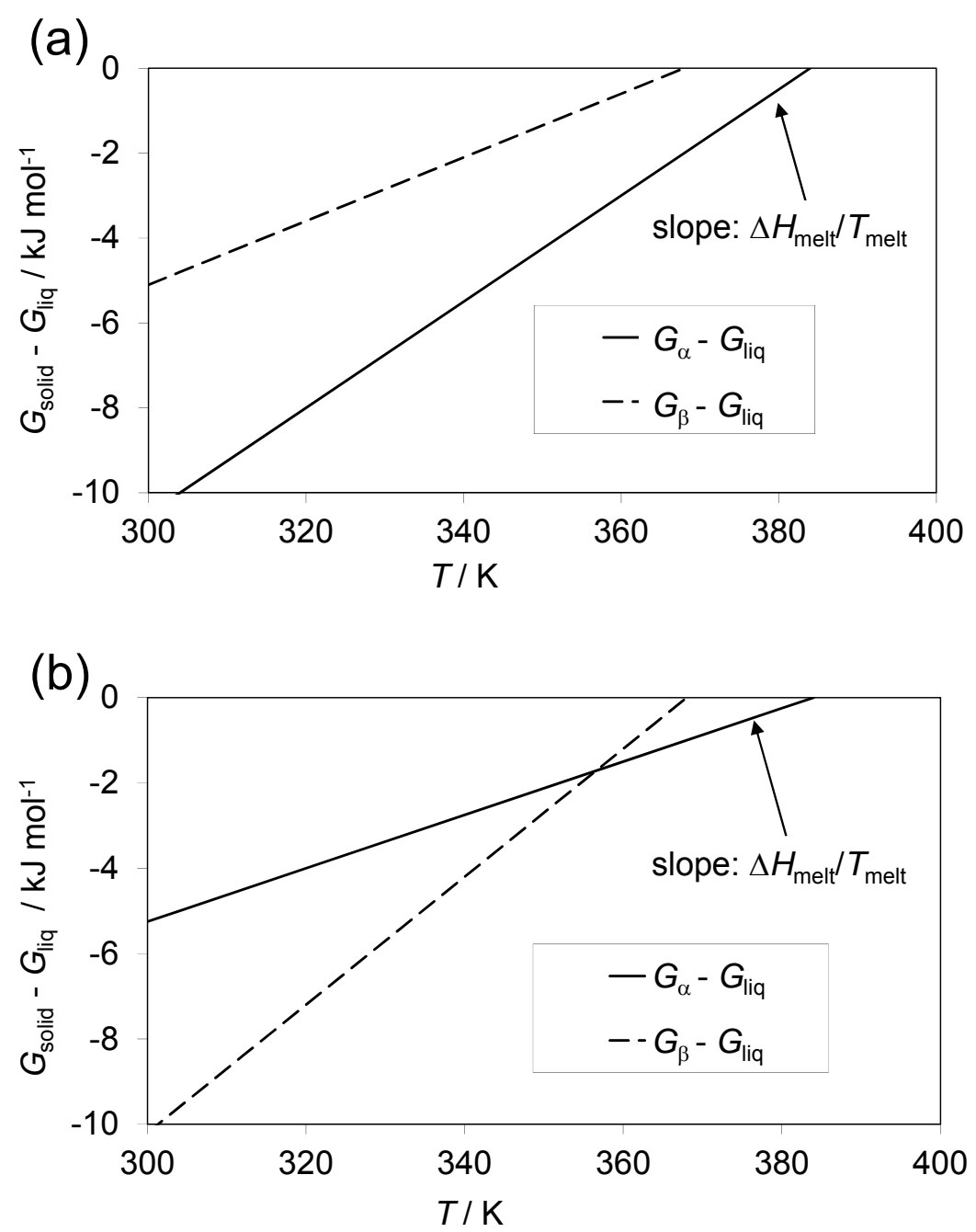

Figure 7.5. Quantitative ET diagram (difference of enthalpy/energy between a polymorph and melt) for (a) a monotropic and (b) an enantiotropic system (heat capacity differences neglected)

It is important, however, to realise that Equation 7.7 neglects the heat capacity difference between the solid and the melt. This can lead to errors in the calculated transition temperature, especially if the melting temperatures of the two polymorphs are far apart, and if the difference between the heat capacities of the melt and polymorph are not negligible, which is often the case. In such a situation, Equation 7.5 should be applied. The effect of heat capacity differences on calculated transition temperatures is demonstrated in Figures 7.6a and 7.6b. For that particular substance, the melting points were $28 \mathrm{~K}$ apart and the heat capacity difference between the melt and the solid was about $0.4 \mathrm{~J} \mathrm{~K}^{-1} \mathrm{~g}^{-1}$. Without taking the heat capacity difference between melt and solid into account, a 
transition temperature of $295 \mathrm{~K}$ was calculated (Figure 7.6a). Considering the measured heat capacities led to a shift of the calculated transition temperature to $340 \mathrm{~K}$ (Figure 7.6b). This value was later confirmed by slurry experiments of the two polymorphs at various temperatures. Performing such slurry experiments is highly recommended to confirm the calculated values of transition temperatures.

(a)

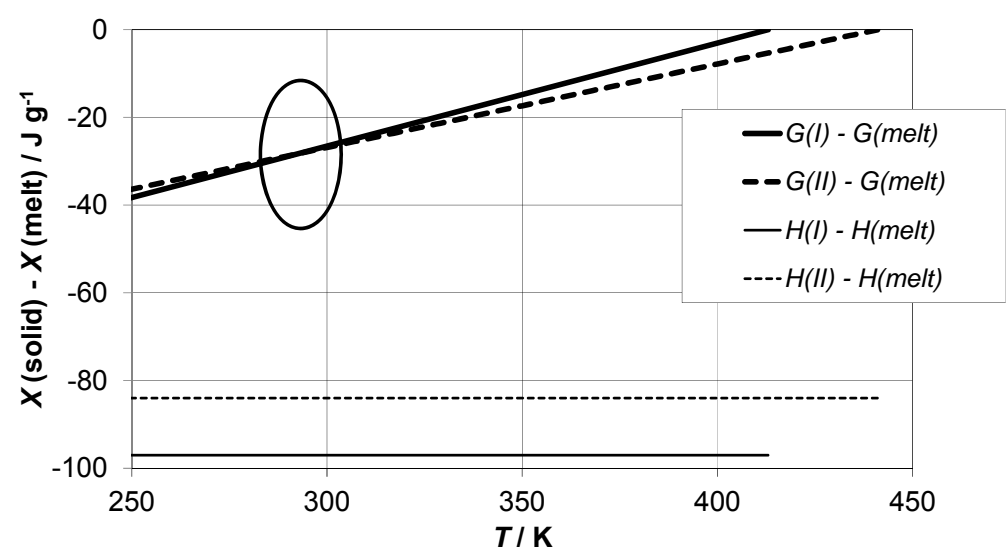

(b)

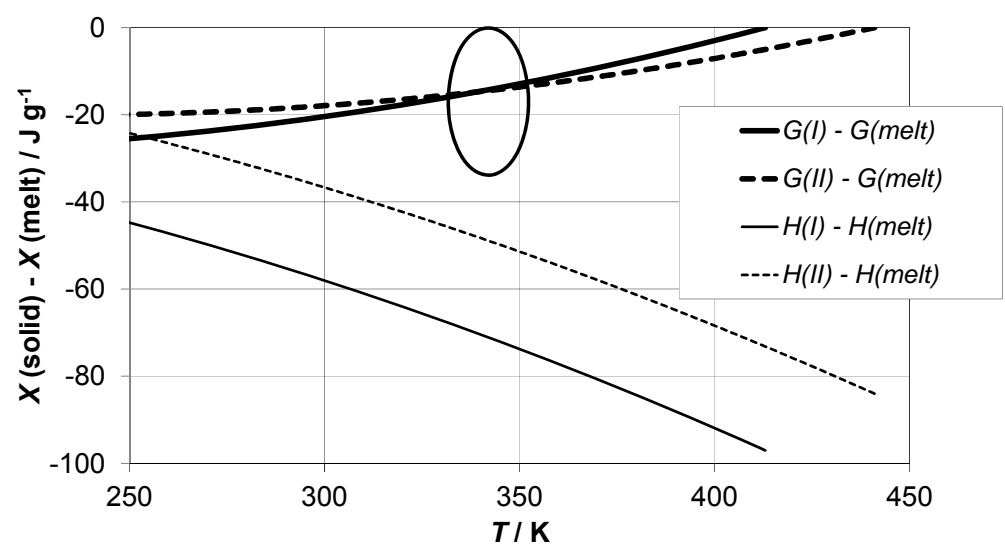

Figure 7.6. Quantitative ET diagram. Example for a certain API: $T_{\text {melt,I }}=413 \mathrm{~K}$, $\Delta H_{\text {melt,I }}=97 \mathrm{~J} \mathrm{~g}^{-1} ; T_{\text {melt,II }}=441 \mathrm{~K}, \Delta H_{\text {melt,I }}=84 \mathrm{~J} \mathrm{~g}^{-1}$. (a) heat capacity difference between the melt and solid neglected, (b) measured heat capacity difference between the melt and solid $\left(c_{\mathrm{p}, \mathrm{I}}-c_{\mathrm{p}, \text { melt }} \approx c_{\mathrm{p}, \mathrm{I}}-c_{\mathrm{p}, \text { melt }} \approx-0.4 \mathrm{~J} \mathrm{~K}^{-1} \mathrm{~g}^{-1}\right)$ taken into account. Note that units are not in mole units but in mass units. This has no effect on Equations 7.4 to 7.7 as long as the same set of units is used throughout

\subsubsection{Hydrates}

As mentioned in Section 7.2, the form chosen for the final drug product is in most cases either the non-solvated polymorph, which is thermodynamically stable at room temperature, or a hydrate. Therefore, hydrates deserve special attention and both their thermodynamic and kinetic properties have to be elucidated. 
Both stoichiometric and non-stoichiometric hydrates exist. In stoichiometric hydrates, the ratio between the number of water molecules and the number of compound molecules in the crystal is well-defined and constant over a certain range of water activities. The water molecules are then normally located at specific positions in the crystal lattice as schematically depicted in Figure 7.1. Nonstoichiometric hydrates, however, contain an amount of water which can continuously vary within a certain range when the water activity of the environment of the crystal is varied. In such cases, the water molecules often reside within channels, larger voids or layers of the crystal lattice [21]. Compounds may also form multiple stoichiometric hydrates. An example is shown in Figure 7.7. This particular substance can exist as a mono-, sesqui-, di-, hemipenta- and trihydrate.

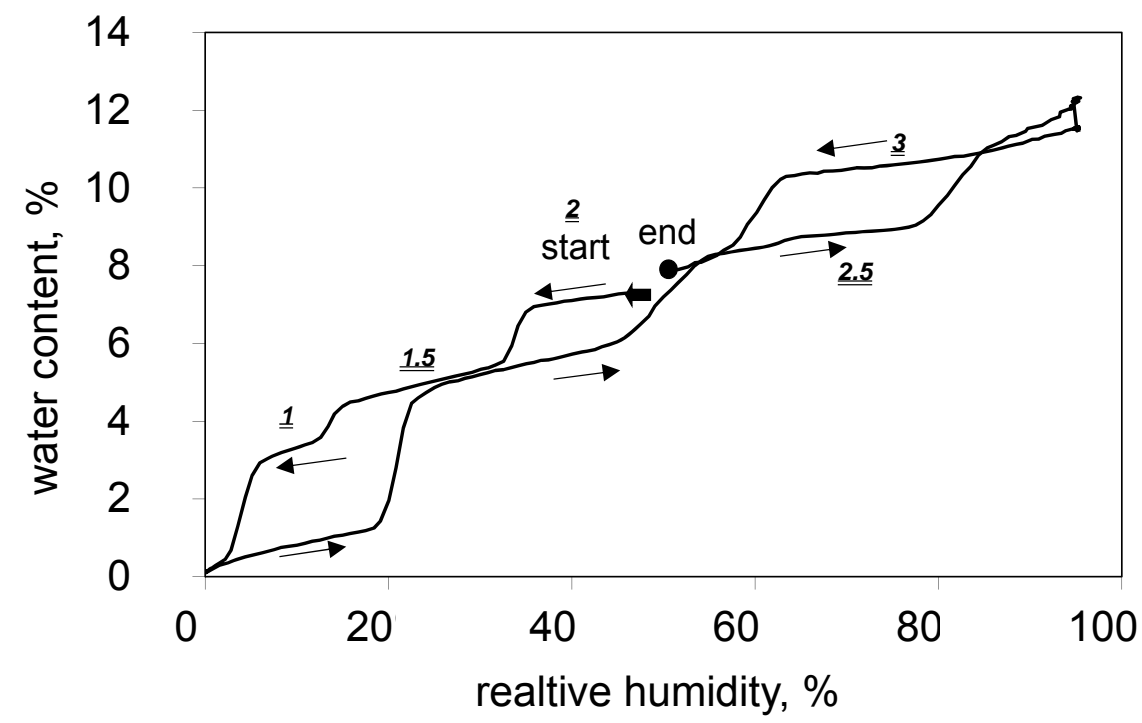

Figure 7.7. Dynamic vapour sorption (DVS) of a substance which can form an anhydrate, as well as a mono-, sesqui-, di-, hemipenta- and trihydrate

While the thermodynamic properties of non-solvated forms depend only on temperature and pressure, the free energy of hydrates (and solvates) is influenced by the water (or solvent) activity of the environment. The formation of a stoichiometric hydrate with $n$ molecules of water per compound molecule can be described by Equation 7.8.

$$
\mathrm{A}(\mathrm{s})+\mathrm{nH}_{2} \mathrm{O} \rightleftarrows \mathrm{A} \cdot \mathrm{nH}_{2} \mathrm{O}(\mathrm{s})
$$

The equilibrium constant $K_{\mathrm{h}}$ for hydrate formation can be expressed by Equation 7.9 , where $a_{\mathrm{H}_{2} \mathrm{O} \text {,eq }}$ is the water activity at equilibrium. The activities of solids are defined as unity and therefore do not appear in Equation 7.9.

$$
K_{\mathrm{h}}=a_{\mathrm{H}_{2} \mathrm{O}, \mathrm{eq}}^{-\mathrm{n}}
$$


Equation 7.9 shows that there is one very well-defined water activity, where the hydrate and anhydrate are in equilibrium. If the water activity (or relative humidity) is slightly below this critical water activity, only the anhydrate is stable, and if it is slightly above, only the hydrate is stable, i.e. the typical situation of a step-wise phase transition is observed. Knowledge of this critical water activity is of great importance in order to be able to decide which form is the preferred one for development.

The standard free energy of hydrate formation can then be calculated using Equation 7.10.

$$
\Delta G_{\mathrm{h}}^{0}=-R T \ln \left(K_{\mathrm{h}}\right)
$$

Knowing $\Delta G_{\mathrm{h}}{ }^{0}$, which can be calculated from the experimentally determined $a_{\mathrm{H}_{2} \mathrm{O} \text {,eq }}$, the free energy difference between hydrate and anhydrate $\left(\Delta G_{\mathrm{h}}\right)$ can be calculated as a function of water activity using Equation 7.11.

$$
\Delta G_{\mathrm{h}}=\Delta G_{\mathrm{h}}^{0}+R T \ln \left(a_{\mathrm{H}_{2} \mathrm{O}}^{-\mathrm{n}}\right)
$$

From the free energy difference of the hydrate and anhydrate, important parameters like the ratio of solubilites $(L)$ and intrinsic dissolution rates $(V)$ between the hydrate and anhydrate can be calculated (Equation 7.12). For the derivation of Equation 7.12, the assumption is made that the activity coefficient is unity. This is generally very good approximation as long as the solubility is not very high.

$$
\Delta G_{\mathrm{h}}=\Delta G_{\mathrm{h}}^{0}+R T \ln \left(a_{\mathrm{H}_{2} \mathrm{O}}^{-\mathrm{n}}\right)=R T \ln \frac{L_{\text {hydrate }}}{L_{\text {anhydrate }}}=R T \ln \frac{J_{\text {hydrate }}}{J_{\text {anhydrate }}}
$$

In water, the water activity is 1 and Equation 7.12 can be simplified obtaining Equation 7.13.

$$
\Delta G_{\mathrm{h}}^{0}=R T \ln \frac{L_{\text {hydrate }}}{L_{\text {anhydrate }}}=R T \ln \frac{J_{\text {hydrate }}}{J_{\text {anhydrate }}}
$$

Combining Equation 7.12 with Equations 7.9 and 7.10 yields Equation 7.14, which describes the relationship between the critical water activity for hydrate formation, solubility and the dissolution rate in water.

$$
\frac{L_{\text {hydrate }}}{L_{\text {anhydrate }}}=\frac{J_{\text {hydrate }}}{J_{\text {anhydrate }}}=a_{\mathrm{H}_{2} \mathrm{O}, \mathrm{eq}}^{\mathrm{n}}
$$

It can be seen that the solubility and dissolution rate of the hydrate in water are smaller compared to the anhydrate if the critical water activity is smaller than 1 . The reduction of solubility and dissolution rate is higher if the critical water activity is lower and if the stoichiometry of the hydrate is higher. 


\subsubsection{Example for the Selection of a Hydrate or Anhydrate}

Carbamazepine (CBZ) can be used as an example how the characterisation and selection process should be carried out. Many solid forms of CBZ are known. In addition to many solvates and co-crystals, four non-solvated forms and a dihydrate are described in the literature. A brief description of the latter is given in Table 1 [22,23]. Different authors use different nomenclature for the forms of CBZ. It is therefore recommended that the crystal system is listed at the same time, since only that is unambiguous.

Table 1. Properties of various forms of carbamazepine (CBZ).

\begin{tabular}{cccccc}
\hline & Form I & Form IIa (IV) & Form IIb & Form III & Dihydrate \\
\hline $\begin{array}{c}\text { Crystal } \\
\text { System }\end{array}$ & triclinic & C-monoclinic & trigonal & P-monoclinic & orthorhombic \\
\hline $\begin{array}{c}\text { Melting } \\
\text { behaviour }\end{array}$ & $\sim 194^{\circ} \mathrm{C}$ & $\sim 188^{\circ} \mathrm{C}$ & $\begin{array}{c}135-170^{\circ} \mathrm{C} \\
\rightarrow \text { Form I }\end{array}$ & $\begin{array}{c}162-175^{\circ} \mathrm{C} \\
\rightarrow \text { Form I }\end{array}$ & n.a. \\
\hline \multirow{2}{*}{ Properties } & $\begin{array}{c}\text { thermodyna- } \\
\text { mically stable } \\
\text { above } \sim 73^{\circ} \mathrm{C}\end{array}$ & & $\begin{array}{c}\text { thermodynam- } \\
\text { ically stable } \\
\text { below } \sim 73^{\circ} \mathrm{C}\end{array}$ & \\
\hline
\end{tabular}

According to what was mentioned above, the two forms which should be considered for development are the thermodynamically stable form at room temperature (Form III) and the dihydrate. In order to be able to make this choice, both the thermodynamics and kinetics of hydrate formation and dehydration have to be elucidated. A dynamic vapour sorption (DVS) experiment on Form III (Figure 7.8) showed no indication of hydrate formation within the timeframe of the experiment.

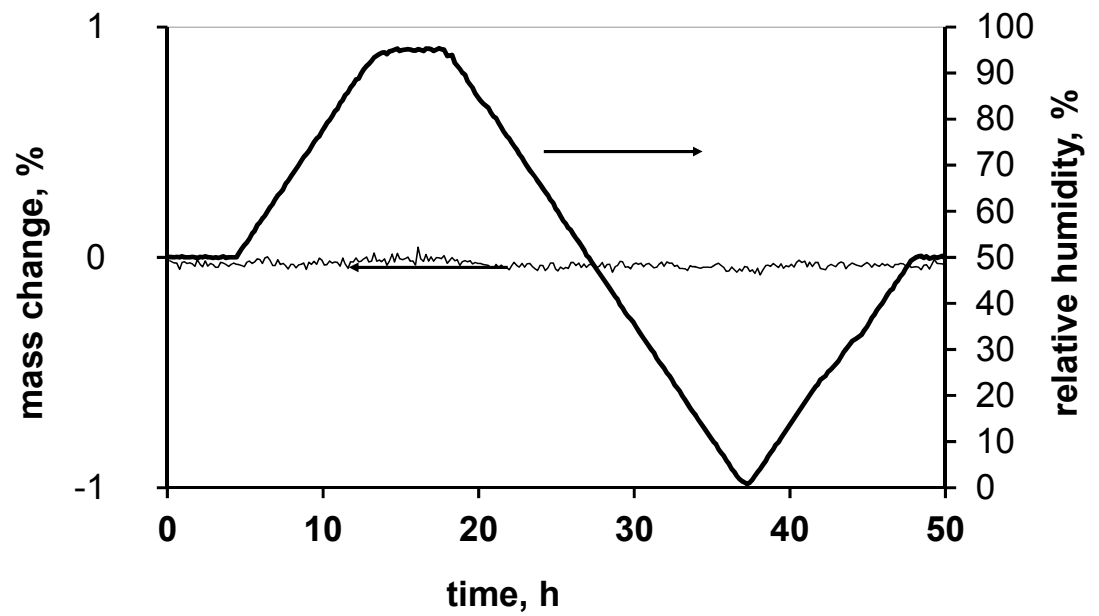

Figure 7.8. DVS of CBZ Form III. The thick black line represents the humidity and the thin black line the mass of the sample. It can be seen that Form III does not convert to the dihydrate, even though it is exposed to humidities higher than $a_{\mathrm{H} 20 \text {,eq }}$ for one day 


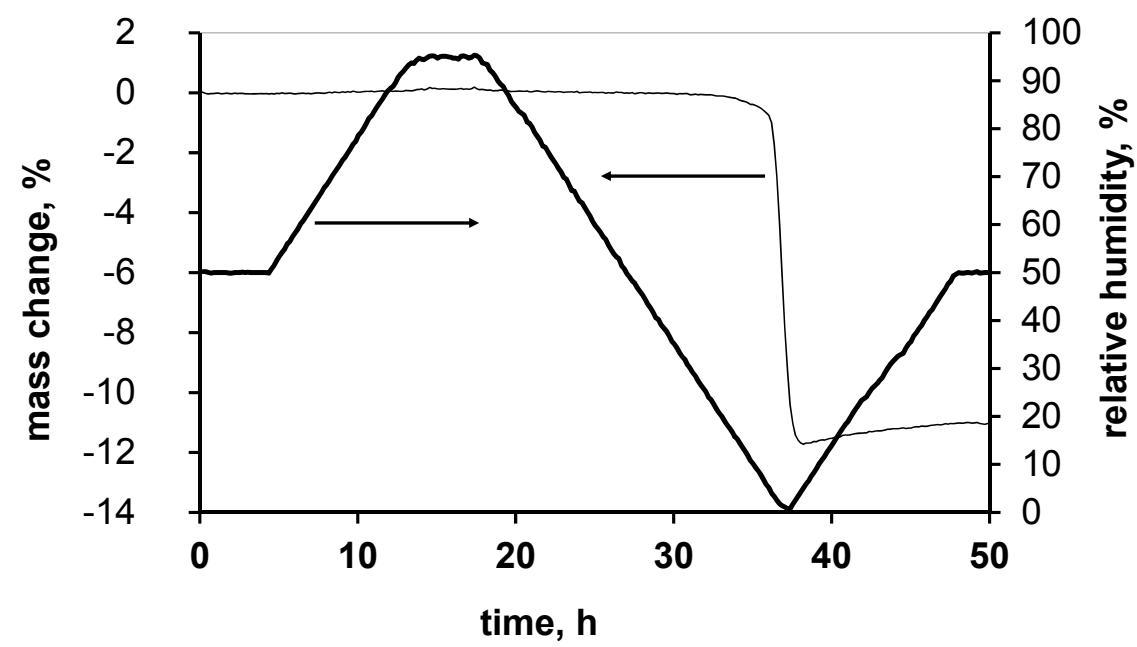

Figure 7.9. DVS of CBZ dihydrate. Water loss is observed at about $5 \%$ relative humidity, which is well below $a_{\mathrm{H} 20 \text {,eq }}$

Figure 7.9 is the corresponding DVS of the dihydrate. It shows that dehydration occurs at about $5 \%$ relative humidity. These two experiments reveal that the critical water activity for the formation of the dihydrate is above 0.05 , but they fail to provide a reasonable estimate for the actual value. This is generally the case for DVS experiments, since hydration and dehydration can be very slow, such that kinetic effects dominate.

An appropriate method to determine the critical water activity is to perform slurry experiments on mixtures of the hydrate and anhydrate in water/solvent mixtures of known water activity. Solvents which do not form solvates with the particular substance should be chosen, so that solvate formation will not compete with the formation of hydrates and non-solvated forms. If the water activity of this solvent/water mixture is now below the critical water activity of hydrate formation, the hydrate will dehydrate and, at the end, a pure anhydrate will be obtained and vice versa. A correction may have to be applied for the water removed from the solvent/water mixture due to hydrate formation or the water released to the solvent/water mixture due to dehydration of the hydrate.

In Figure 7.10, the resulting Raman spectra of another substance which can form an anhydrate and a hemihydrate in slurries of various water activities are shown. It can clearly be seen that the characteristic peak of the hemihydrate is observed at water activities of 0.8 and higher, i.e. the critical water activity for hemihydrate formation is about 0.75 in that particular case.

Figure 7.11 shows the result of such slurry experiments of CBZ Form III and the dihydrate in a variety of water/solvent mixtures. It shows that the critical water activity for dihydrate formation is about 0.65 at $23^{\circ} \mathrm{C}$. Within experimental error, 
it is independent of the solvent, as it should be. Using this value, the standard free energy difference between the dihydrate and Form III can be calculated as -2.4 $\mathrm{kJ} / \mathrm{mol}$ (Equations 7.9 \& 7.10). From this, it follows that Form III is about 2.6 times more soluble in water than the dihydrate.

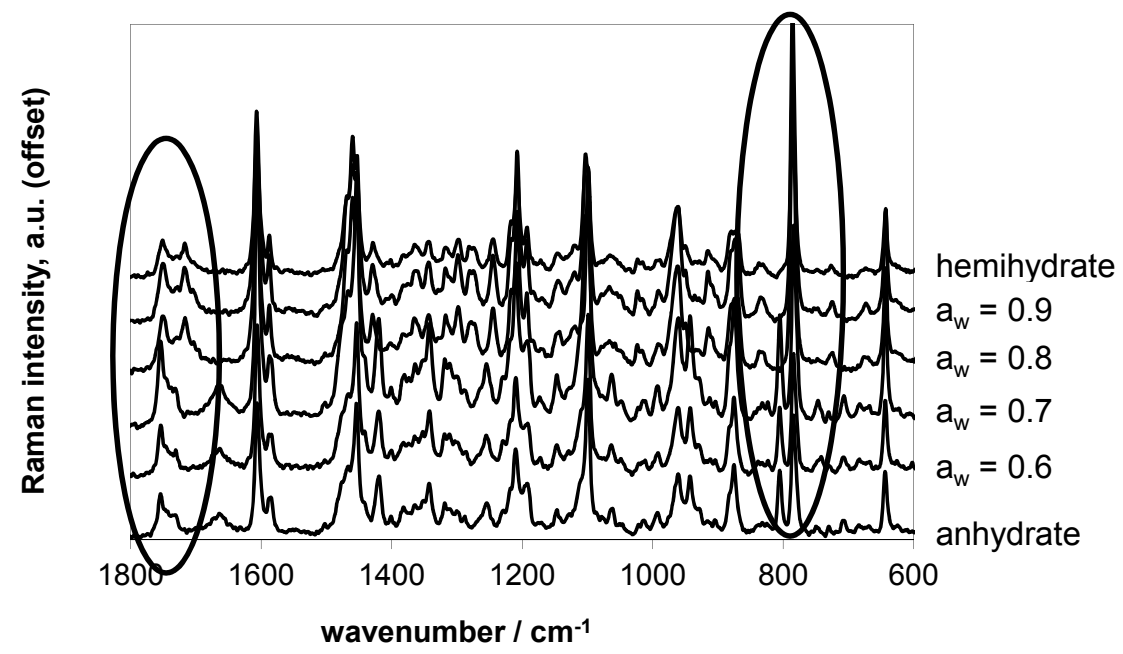

Figure 7.10. Raman spectra of the anhydrate (bottom) and hemihydrate (top) of an API. The spectra in between were recorded after mixtures of the anhydrate and hemihydrate were slurried for two days in water/solvent mixtures with water activities of 0.6, 0.7, 0.8 and 0.9. It can be seen that for $a_{\mathrm{w}}=0.8$ and 0.9 , complete conversion of the anhydrate to

the hemihydrate occurred, while the opposite was the case for $a_{\mathrm{w}}=0.7$ and 0.6

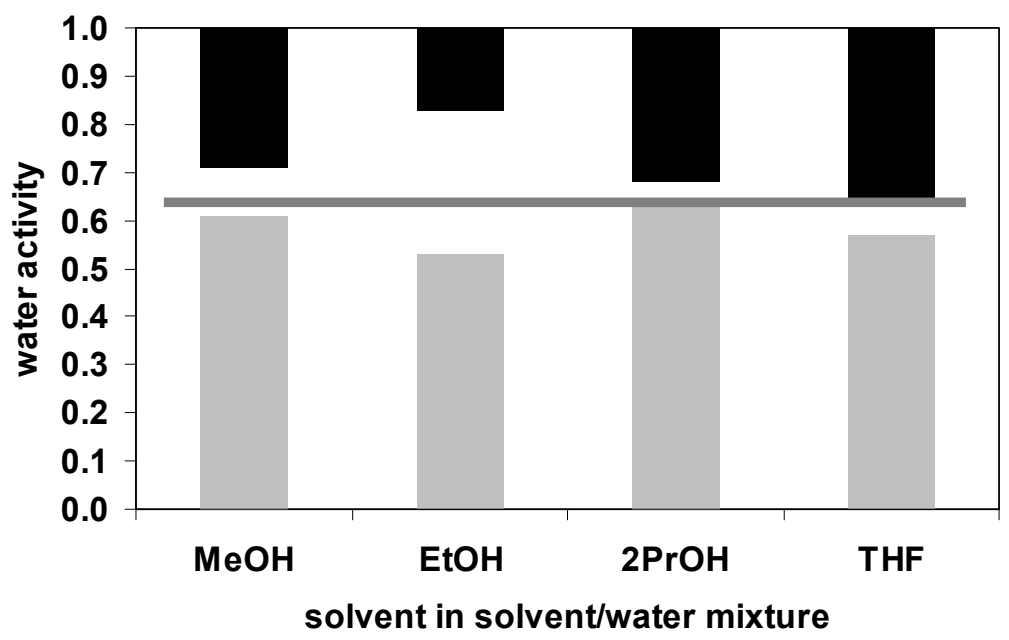

Figure 7.11. Results of slurry experiments of mixtures of CBZ Form III and the dihydrate in various water/solvent mixtures with water activities of $0.1,0.2,0.3$, etc.

$\left(\mathrm{T}=23^{\circ} \mathrm{C}\right)$. Grey denotes that the resulting form was Form III, black denotes that the resulting form was the dihydrate. ( $\mathrm{MeOH}$ - methanol, EtOH - ethanol, 2PrOH - 2-propanol, THF - tetrahydrofuran) 
It has to be considered that the critical water activity for hydrate formation is also temperature dependent and increases with temperature in the vast majority of cases. At $37{ }^{\circ} \mathrm{C}$, the corresponding values for CBZ are: $a_{\mathrm{H} 2 \mathrm{O} \text {, eq }} \approx 0.8, \Delta G_{\mathrm{h}}{ }^{0} \approx-1.5$ $\mathrm{kJ} / \mathrm{mol}$, solubility ratio in water $\approx 1.8$. Based on these data gathered for the two forms of CBZ, Form III would be favoured, since

- it has an approximately two-fold higher water solubility and CBZ is BCS Class II, i.e. solubility is a bottleneck

- it is thermodynamically stable in dry conditions and up to fairly humid conditions (65 \% r.h.)

- if it is exposed to high humidities for many hours, it will still be kinetically stable

Of course, other aspects which might affect the ease of manufacturing, processing and formulation have to be taken into account as well.

Knowledge of the critical water activity as a function of temperature is also very helpful for the design of the crystallisation process of Form III, since a water activity of the crystallisation medium exceeding this critical water activity should always be avoided.

\subsection{FORM SCREENING}

Clearly, before being able to select the optimal form, the relevant forms have to be identified and prepared, i.e. form screening has to be carried out. Most substances will be able to form salts, co-crystals or both, and therefore salt or cocrystal screening is often the first step in form selection.

\subsubsection{Screening for Salts/Co-Crystals}

In general, salt formation will be possible if the molecule contains acidic or basic groups, which is the case for a large fraction of active molecules. Since making a salt will normally involve an additional step in the synthesis and since the molecular weight of a salt will always be higher than that of the neutral molecule, salts will only be chosen if they promise to have clear advantages compared to the free acid/base. As a rule, a salt is chosen if the free acid/base has at least one of the following undesirable properties:

- very low solubility in water,

- low melting point,

- high hygroscopicity,

- low chemical stability,

- problematic solid forms, such as channel hydrates or enantiotropic polymorphs with a transition temperature close to room temperature,

- other undesirable properties such as unfavourable habit,

- intelectual property (IP) issues. 
The potential acids or bases which might be able to form a salt with the API under investigation are chosen based on $\mathrm{p} K_{\mathrm{a}}$ differences, counterion toxicity (preferably Generally Recognized As Safe (GRAS)) status, see also the classification scheme in [4]), etc. As a rule of thumb, a $\mathrm{p} K_{\mathrm{a}}$ difference of 2-3 is sufficient for salt formation. It is advisable to perform salt screening and salt selection in stages, starting on the microscopic scale (e.g. a 96-well plate format) where a large number of salts is produced and characterised using a limited number of methods (e.g. birefringence, Raman spectroscopy, X-ray powder diffraction (XRPD)) in order to identify a few promising candidates. Suitable methods for producing salts in such a high-throughput format include very slow evaporation of stoichiometric quantities of the acid and base in a variety of solvents where the solubilities of the acid and the base are sufficiently high, and slurrying of the acid and base in solvents where the solubility of the salt is not very high. In the second stage, the successful candidates of the high-throughput screening are then produced on the scale of a few hundred milligrams and characterised in more detail in order to select the best ones. Desirable properties of the salts include crystallinity, high water solubility, low hygroscopicity, good chemical stability and a high melting point. The relative importance of these properties may vary from project to project.

Co-crystals can offer valuable alternatives, especially for very weak bases or acids. Reasons for attempting to make co-crystals are the same as for salts.

\subsubsection{Example of a Co-Crystal Screen}

A certain API had a needle-like morphology with an extremely large aspect ratio (Figure 7.12). Due the corresponding low bulk density, this posed considerable problems during formulation and processing, since the API required a high dosage strength. Attempts to change the morphology by changing crystallisation conditions and the solvents for crystallisation failed, and salts were not an option, because the API was neither acidic nor basic. Therefore, co-crystal formation was attempted.

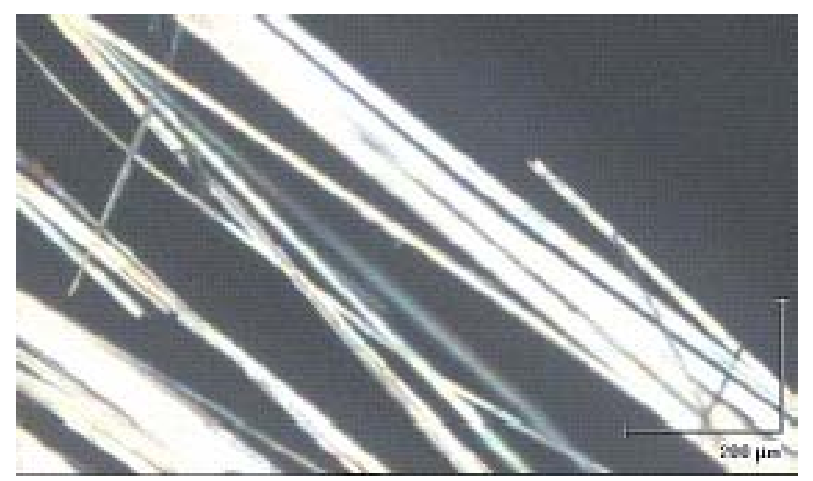

Figure 7.12. Microscopic image of an API with an unfavourable needle-like morphology. 
The API had an amide group which can form very strong interactions with carboxylic acids by forming eight-membered rings with two hydrogen bonds (see Figure 7.13), making carboxylic acids promising candidates for co-crystal formation.

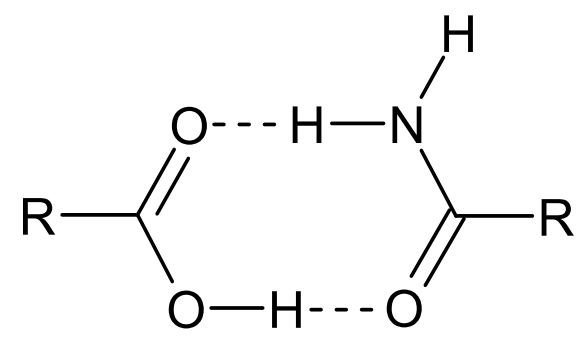

Figure 7.13. Possible interaction motif between an amide and a carboxylic acid.

The following 24 compounds were selected as potential co-crystal formers based on their potential interaction with the API and their toxicity:

- acetylsalicylic acid

- $\quad$-aspartic acid

- benzenesulphonic acid

- benzoic acid

- citric acid

- ethanedisulphonic acid

- fumaric acid

- gentisic acid

- $\quad$-lactic acid

- maleic acid

- L-malic acid

- malonic acid

- $\quad L$-mandelic acid

- methanesulphonic acid

- oxalic acid

- pamoic acid

- saccharin

- succinic acid

- L-tartaric acid

- ethyl maltol

- methyl 4-hydroxybenzoate

- urea

- $\quad L$-proline

- $L$-tryptophan 
In order to optimise the chances of success of a co-crystal screen, an understanding of phase diagrams of the co-crystal former, API and solvent is very beneficial $[24,25]$. Depending on the respective behaviour, different strategies are optimal.

In this particular case, equimolar amounts of the API and co-crystal former were mixed in four different solvents where the API and co-crystal former had similar solubilities. These $96(4 \times 24)$ solutions were then evaporated very slowly under a controlled nitrogen stream in a 96-well plate. The resulting solids were analysed by Raman spectroscopy and XRPD. In a second set of experiments, equimolar amounts of the API and co-crystal former were mixed in four different solvents which did not dissolve the API and co-crystal former. These slurries were temperature-cycled for 4 days, then the solvent was evaporated and the solids were analysed in the same way. The XRPD and Raman spectra of the 192 samples showed that, in many cases, a crystalline API and/or crystalline co-crystal former was obtained in the end. For benzoic, ethanedisulphonic, maleic, $L$-malic, $L$ tartaric acid and ethyl maltol, Raman and XRPD spectra were recorded which differed from the components, i.e. they were promising candidates for co-crystal formation (see also Section 7.4.2). These were then scaled-up to about $100 \mathrm{mg}$ and first characterised by Raman spectroscopy and XRPD in order to verify that the same form was obtained in the scale-up experiment as in the microscopic high-throughput experiment. They were further characterised by NMR, elemental analysis, differential scanning calorimetry (DSC), thermogravimetry-Fourier transform IR (TG-FTIR), DVS, aqueous solubility and light microscopy in order to judge if their physical properties were favourable. It turned out that co-crystals with benzoic acid and maleic acid had good physical properties (high melting point, low hygroscopicity and sufficient aqueous solubility) and fulfilled the target of the study, i.e. they had a much more desirable morphology.

\subsubsection{Screening for Polymorphs}

Screening for polymorphs and other relevant solid forms is not an easy task and requires a lot of experience, since it has to be ensured that the largest possible variety in crystallisation conditions is used, both in terms of the type of crystallisation methods and solvent choice. The identification of solvates is also important, not because they might be used in the dosage form, but because it is advisable not to use solvents which can form solvates with a particular compound in a crystallisation process to produce that compound.

The fact that different polymorphic forms can be obtained when the crystallisation conditions are changed can be explained by Ostwald's rule of stages which states that, "When leaving a given state and in transforming to another state, the state which is sought out is not the thermodynamically stable one, but the state nearest in stability to the original state." [26]. The rule implies that during a crystallisation process, several forms may crystallise in sequence starting from the least stable form and ending up in the stable form. Or, in other words, if a very fast crystallisation method, such as a rapid precipitation, is applied, one is likely to obtain a metastable form, while a very slow 
crystallisation process, such as a slow cooling or slurry ripening, would yield the stable form. But, true to its name, Ostwald's rule of stages is a rule and not a law, and many violations of this rule have been reported. Nevertheless, it is a useful concept for the design of polymorphism screens.

A more mechanistic view of the formation of different polymorphs takes into account cluster formation, nucleation and crystal growth $[27,28]$. This model also readily explains the influence of the solvent (if crystallisation is performed from a solution) and additives in addition to the crystallisation speed. Clusters and nuclei are small, and therefore the surface free energy significantly influences the value of the total free energy of the cluster or nucleus. This is depicted schematically in Figure 7.14. The free energy of individual molecules, i.e. very small clusters, is equal to the free energy in solution $\left(G_{\text {solution }}\right)$. Due to the surface energy, the free energy then increases with increasing cluster radius up to a critical cluster radius $\left(r^{*}\right)$ and the corresponding free energy ( $G_{\text {nucleus }}$ ). Further growth of the nucleus leads to a decrease in free energy and is therefore spontaneous. When the cluster is large enough such that the surface energy becomes negligible, the free energy of the crystal $\left(G_{\alpha}\right.$ or $\left.G_{\beta}\right)$ is adopted, which is smaller than $G_{\text {solution }}$ if the solution is super-saturated. Solvent and/or additive molecules can preferentially adsorb on the surface of the nuclei and can therefore alter the heights of the activation barriers $\Delta G_{\alpha}{ }^{*}=G_{\text {nucleus, } \alpha}-G_{\text {solution }}$ and $\Delta G_{\beta}{ }^{*}=$ $G_{\text {nucleus }, \beta}-G_{\text {solution }}$ for polymorph $\alpha$ and $\beta$, respectively. Since the surfaces of different polymorphs may be different, this preferential adsorption may also depend on the polymorph. Therefore, a situation may be encountered where $\Delta G_{\alpha}{ }^{*}$ is larger than $\Delta G_{\beta}{ }^{*}$ in a certain solvent $\mathrm{A}$, while in another solvent $\mathrm{B}, \Delta G_{\beta}{ }^{*}$ is larger than $\Delta G_{\alpha}{ }^{*}$, such that polymorph $\beta$ is kinetically favoured in solvent A while polymorph $\alpha$ preferentially forms in solvent B. A type of additive that can have a particularly large effect is impurities [29-31] with a similar structure to the main component, since they may adsorb very strongly on the surface of certain nuclei due to their chemical similarity. Such impurities are often formed during the final steps of the synthetic process. It is therefore crucial to perform crystallisations with a variety of solvents in order to increase the chance of successful competition of solvent molecules with impurity molecules for preferential adsorption.

The relative thermodynamic stability of polymorphs of non-solvated forms, on the other hand, is independent of the solvent it is formed in, since the solvent can only contribute to the surface free energy of the crystal. While the surface free energy is an important contribution to the total free energy of nuclei which are less than a few nanometres in diameter, the surface free energy is negligible compared to the bulk free energy if the crystal size is larger than about $100 \mathrm{~nm}$. The formation and stability of solvates, however, is of course influenced by the choice of solvent. This is yet another reason why various solvents should be used in a polymorph screen. 
Therefore, in order to find all possible polymorphs and solvates, a large number of crystallisation methods in a large number of diverse environments have to be carried out.

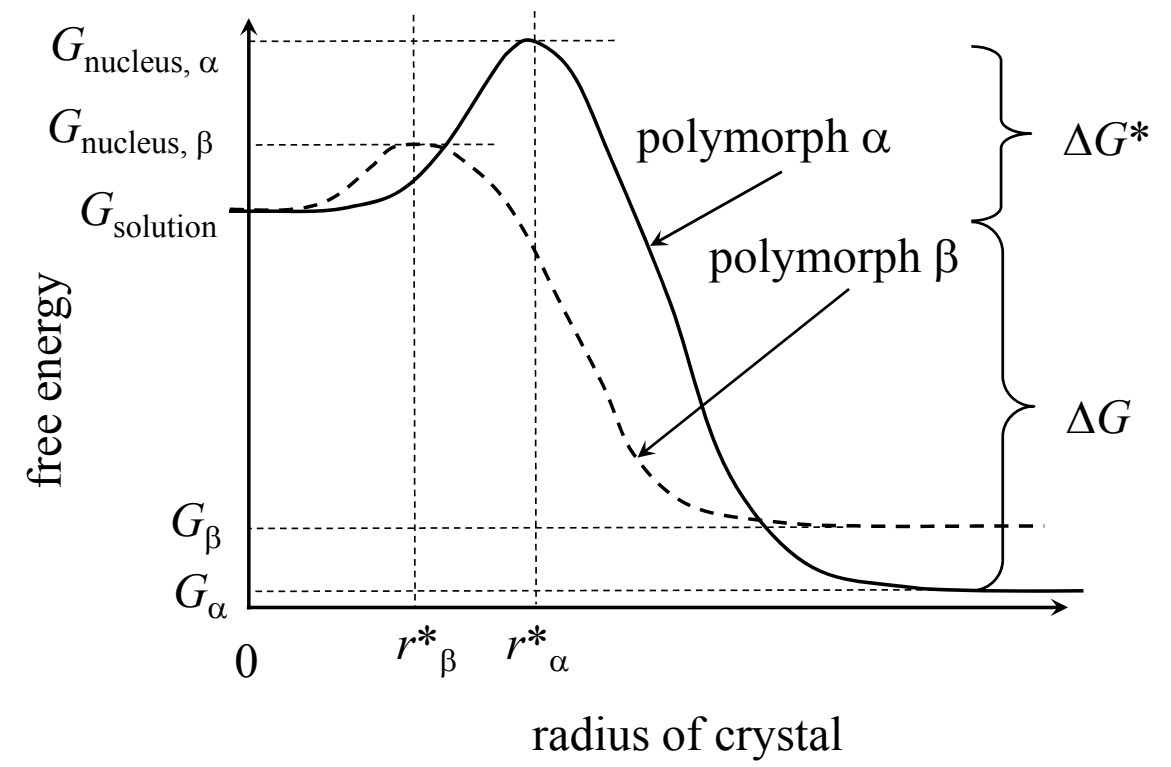

Figure 7.14. Energy barriers for polymorph crystallisation

Classical crystallisation methods have been reviewed by Guillory [32] and are the following:

- crystallisation by cooling a solution,

- crystallisation by evaporation,

- crystallisation by precipitation,

- crystallisation by vapour diffusion,

- suspension equilibration (often also called "slurry ripening"),

- crystallisation from the melt,

- heat induced transformations,

- crystallisation by sublimation,

- desolvation of solvates,

- crystallisation by salting out,

- crystallisation by $\mathrm{pH}$ change,

- lyophilisation.

Within these methods, further diversity can be introduced by changing solvents, temperatures, pressure, rates of cooling, evaporation, etc. Several types of crystallisation experiments are also influenced by the initial solid form that is used (i.e. polymorph, solvate, hydrate or the amorphous form) as this can affect the solubility and hence the degree of supersaturation. As explained above, the choice of solvent is of particular importance. First, it has to be chosen such that the solubility is in a suitable range for the selected type of crystallisation 
experiment (reasonably high solubility for cooling experiments, very low solubility in solvents used for precipitation, etc.). Second, it is important to use solvents with diverse physical properties in order to explore the entire parameter space of possible environments. There are several approaches in order to classify solvents in terms of dissimilarity. A very useful one is the method applied by Gu et al. [33]. They examined 96 solvents in terms of eight relevant solvent properties: hydrogen bond acceptor propensity, hydrogen bond donor propensity, polarity/dipolarity, dipole moment, dielectric constant, viscosity, surface tension and cohesive energy density and sorted the 96 solvents into 15 groups according to their statistical similarity using principal component analysis. As expected, solvent molecules with the same functional groups were often found within the same group. In a thorough polymorphism screen, where the highest diversity is desired, crystallisations using at least one solvent of each group should therefore be carried out, so that the whole spectrum of solvent properties is used in the crystallisation experiments.

Of course, one can never be sure that one has found all relevant polymorphs unless one carries out an infinite number of experiments. This essentially paraphrases McCrone's famous statement, "It is at least this author's opinion that every compound has different polymorphic forms and that, in general, the number of forms known for a given compound is proportional to the time and money spent in research on that compound" [1].

It may also be difficult to reproduce the crystallisation of a metastable form and there are accounts of so-called "disappearing polymorphs", i.e. forms which could be obtained at some stage but not crystallised at a later date. As nucleation is a stochastic process, it is possible that two crystallisation experiments which are performed in exactly the same way may lead to a different outcome. If conditions are chosen properly, however, and any potential seeds of other forms are carefully removed, it should always be possible to reproduce any form if a large enough number of experiments is performed.

\subsubsection{Types of polymorph screens}

Given the fact that it is likely that the more solid forms are found, the more effort is invested in the search and that one can never be sure that all relevant forms are experimentally discovered, two questions arise immediately, i.e. how extensive a search should be carried out and when in the development process a polymorph screen should be carried out. The answers to these questions must take both economic and safety criteria into account.

Regulatory authorities have set some criteria in order to ensure the safety and efficacy of drug products. Therefore, ICH guidelines [2] demand that a polymorph screen has to be carried out in order to obtain regulatory approval for pharmaceutical products. There is, however, no guideline given on how such a screen should be performed. 
Economic criteria would suggest that a polymorph screen is conducted late in the development process of a drug, since the attrition rate of drugs during clinical development is large. Only on the order of $10 \%$ of the drugs entering Clinical Phase I will gain final approval as drug products [34]. On the other hand, it would be an unacceptable risk to develop a dosage form without knowing which form is thermodynamically stable under certain conditions of temperature and humidity. Therefore, a good strategy which balances economic considerations and risk is to carry out several polymorph screens during the development process which are appropriate for the corresponding phase.

Often, the formulation used in Clinical Phase II is close to the final formulation of the drug. Therefore, by that time, the optimal solid form should have been identified with a reasonably high probability. A limited polymorph screen in the preclinical phase or Clinical Phase I will therefore generally have the aim of identifying the thermodynamically stable polymorph and hydrates with a confidence level of $70-90 \%$. If the drug is successful in Clinical Phase II, this confidence level should be increased and the search should be extended to metastable forms, since they may be both important for the development of a crystallisation process of the desired stable form and they may also improve the patent protection of the drug. The relevance of metastable forms for the development of a crystallisation process stems from the fact that metastable forms might be produced inadvertently if their existence and their properties are not known and no appropriate measures are taken so that they are not formed.

A polymorph screen should proceed in a well-structured and standardised way, while recognising that the procedure has to be tailored with respect to the substance under investigation. An approach that is not adapted to the substance is prone to inefficiency and overlooking relevant forms. The procedure used should also be tunable to permit various degrees of thoroughness as stated above.

In essence, a polymorph screen should at least contain the following elements:

- Characterisation of the starting material by methods such as powder or single-crystal XRD, DSC, TG-FTIR or thermogravimetry-mass spectrometry (TG-MS), DVS, Raman or IR, magic-angle spinning (MAS) NMR, solubility measurements, microscopy and HPLC (purity).

- If the substance does not degrade at the melting point, hot-stage microscopy or hot-stage Raman microscopy can be a very efficient way for creating other polymorphs [35].

- Crystallisation experiments from solution, using several techniques with a variety of solvents and solvent mixtures. Due to the practical relevance of hydrates, water and water/solvent mixtures should always be included. Suspension equilibration and slow cooling experiments have particular importance in finding the thermodynamically stable form, which is often the most important aim of a polymorph screen. 
- All new forms have to be characterised by the methods used to characterise the starting material and their thermodynamic relationships have to be elucidated.

- Other techniques such as desolvation of solvates, mechanical stress (pressure, grinding) are necessary to obtain the information needed for the processing and manufacturing steps.

Very useful tools for polymorphism (and salt and co-crystal) screens are highthroughput screening systems, where crystallisation experiments are carried out in an array format, using e.g. 96-well plates. Such systems have been developed by research foundations [36], big pharmaceutical companies [37-39] and companies specialising in solid-state research and development [40-44]. They commonly allow crystallisation by cooling, evaporation, precipitation and slurry conversion. Primary characterisation of the solid is generally performed by Raman microscopy and/or XRPD.

High-throughput screens should always be complemented by larger scale experiments, however, where more of the corresponding form is produced in order to allow a full physicochemical characterisation. It may also be necessary to subject the substance to conditions which are not accessible in a high-throughput screening system.

\subsection{CHARACTERISATION}

Many physicochemical and physical methods are suitable to characterise solids. They include thermal methods like DSC, TG, thermo-mechanical analysis (TMA), thermally stimulated current (TSC), microcalorimetry and thermomicroscopy; spectroscopic methods like IR, Raman, near IR (NIR), NMR and Terahertz spectroscopy; coupled methods like TG-FTIR, TG-MS and hot-stage Raman microscopy; scattering methods like XRD; particle sizing methods like Fraunhofer diffraction, sieving, etc.; and mechanical tests to assess hardness, flow properties, etc.

Other chapters describe many of these important methods in detail (thermal methods which are extremely useful for understanding the thermodynamic properties of solids in Chapter 8, NIR spectroscopic method in Chapter 9). Therefore, we will focus here on only two very important methods for the characterisation of solid forms. Both X-ray diffraction and Raman spectroscopy are very sensitive and specific for the identification of different solid forms.

\subsubsection{X-ray diffraction (XRD)}

The physical basis of both single crystal X-ray diffraction and XRPD is Bragg's law (Equation 7.15), which describes the relationship between the angle for constructive interference $(\Theta)$, layer spacing $(d)$, wavelength $(\lambda)$ and order of the interference $(n)$.

$$
n \lambda=2 d \sin (\Theta)
$$




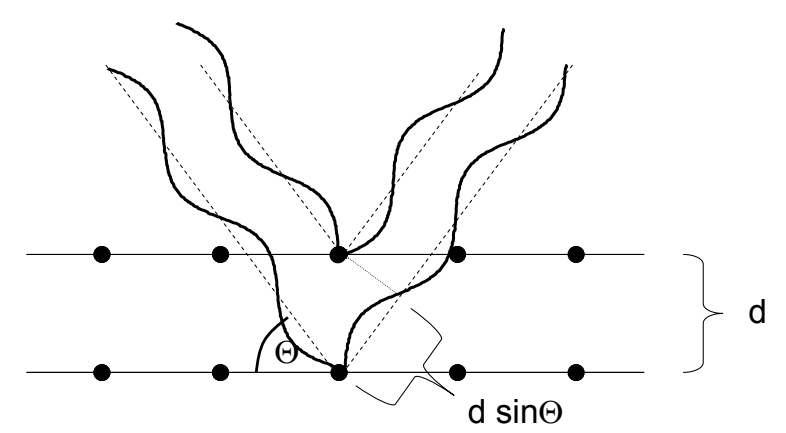

Figure 7.15. Principle of Bragg's law: constructive interference when the path difference is $\lambda$ or multiples of $\lambda$

In single crystal XRD, the crystal is placed in an X-ray beam and the spatial intensity of the diffracted X-ray light is recorded while the angular position of the single crystal is varied. This allows the determination of the crystal structure, i.e. assignment of the space group and location of all atoms in the unit cell.

In XRPD, the sample exposed to the X-ray beam is a powder, and if the particles are randomly oriented, all crystal planes in the crystal lattice will be suitably oriented with respect to the X-ray beam (see Figure 7.15) and give rise to constructive interference at the respective angle. Therefore, a pattern in the form depicted in Figure 7.16 will be obtained. Different polymorphs will have different crystal plane spacings and will therefore lead to a different XRPD patterns (see Figure 7.16). Rare exceptions exist where the differences are so small that they may not be discernible using standard instrumentation.

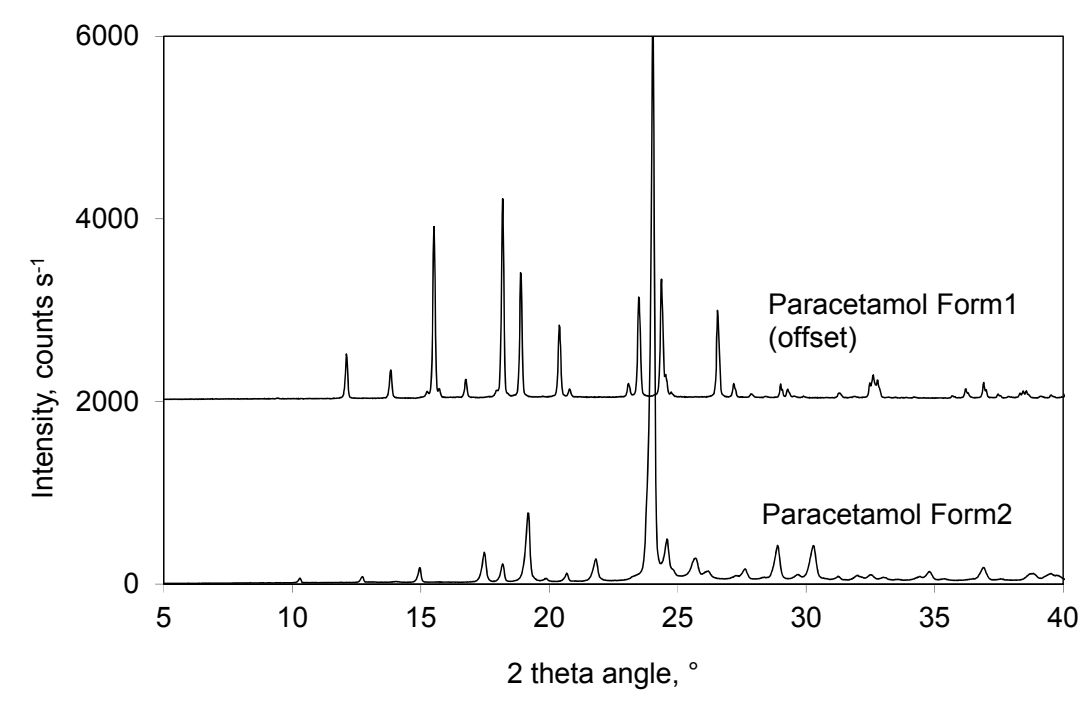

Figure 7.16. XRPD patterns of paracetamol Form 1 (top, offset) and Form 2 (bottom) 
An experimental problem with XRPD is so-called preferential orientation. If the particles are non-spherical (e.g. plates or needles), they may not be randomly oriented. In that case, some lines in the XRPD may become greatly attenuated or even disappear. When comparing the XRPD patterns of various samples, good sample preparation is therefore essential. Orientation effects can, for example, be reduced by gentle grinding of the substance. Investigating the sample by optical microscopy may also be very helpful to see if the particles are preferentially oriented. When a sample is ground, care must be taken that the solid form is not changed due to the grinding. The absence of such changes can be confirmed if the positions of the lines in the XRPD diagram are not shifted by grinding when the intensity of the grinding is varied.

Due to the physical principle of XRD, layer spacings in the crystal are measured and the signal will be the stronger when more layers with a constant spacing are present in the solid, i.e. the farther the spatial order extends. XRPD is therefore sensitive to long range order.

\subsubsection{Raman spectroscopy}

Like IR and NIR, Raman is a vibrational spectroscopy technique. Vibrational spectra are mainly governed by vibrations within the molecule, but since these vibrations are influenced by the environment of the molecule and hence the crystal packing, they will also differ for different polymorphs of the same molecule. In contrast to X-ray diffraction, these techniques primarily probe short range order.

Raman and IR are complementary methods. They differ in their selection rules (change of dipole moment or polarisability during vibration) and in practical aspects. The main advantages of Raman compared to IR in connection with solidstate characterisation are:

- no sample preparation is necessary (IR often uses $\mathrm{KBr}$ pellets; modern attenuated total reflectance IR (ATR-IR) also eliminates sample preparation),

- in general, narrower bands are found,

- Raman microscopy requires less sample preparation and has better spatial resolution than IR-microscopy,

- Raman spectroscopy can easily be applied for online monitoring (see Chapter 9),

- low frequencies are more readily attainable (this is the range of lattice vibrations, where the difference between polymorphs if often very pronounced),

- measurements can be performed through glass/quartz containers,

- little interference from water (weak Raman scatterer).

The advantages of IR are lower instrument cost, wider availability and no problems with samples that exhibit fluorescence. 
Given the advantages of Raman spectroscopy, the focus of this section is placed on that technique. Compared to XRPD, differences in the Raman spectra of two polymorphs will in general not be as obvious, since the spectra are basically determined by the structure of the corresponding molecule. Nevertheless, the specificity of spectroscopic and diffraction methods with respect to the differentiation of polymorphs is similar if a high quality Raman spectrometer with very good wavenumber resolution is used.

In Figure 7.17a, the Raman spectra of three forms of CBZ are depicted (see Table 1). While the spectra look essentially similar, clear line shifts can, for example, be observed between 1550 and $1650 \mathrm{~cm}^{-1}$ (Figure 7.17b).
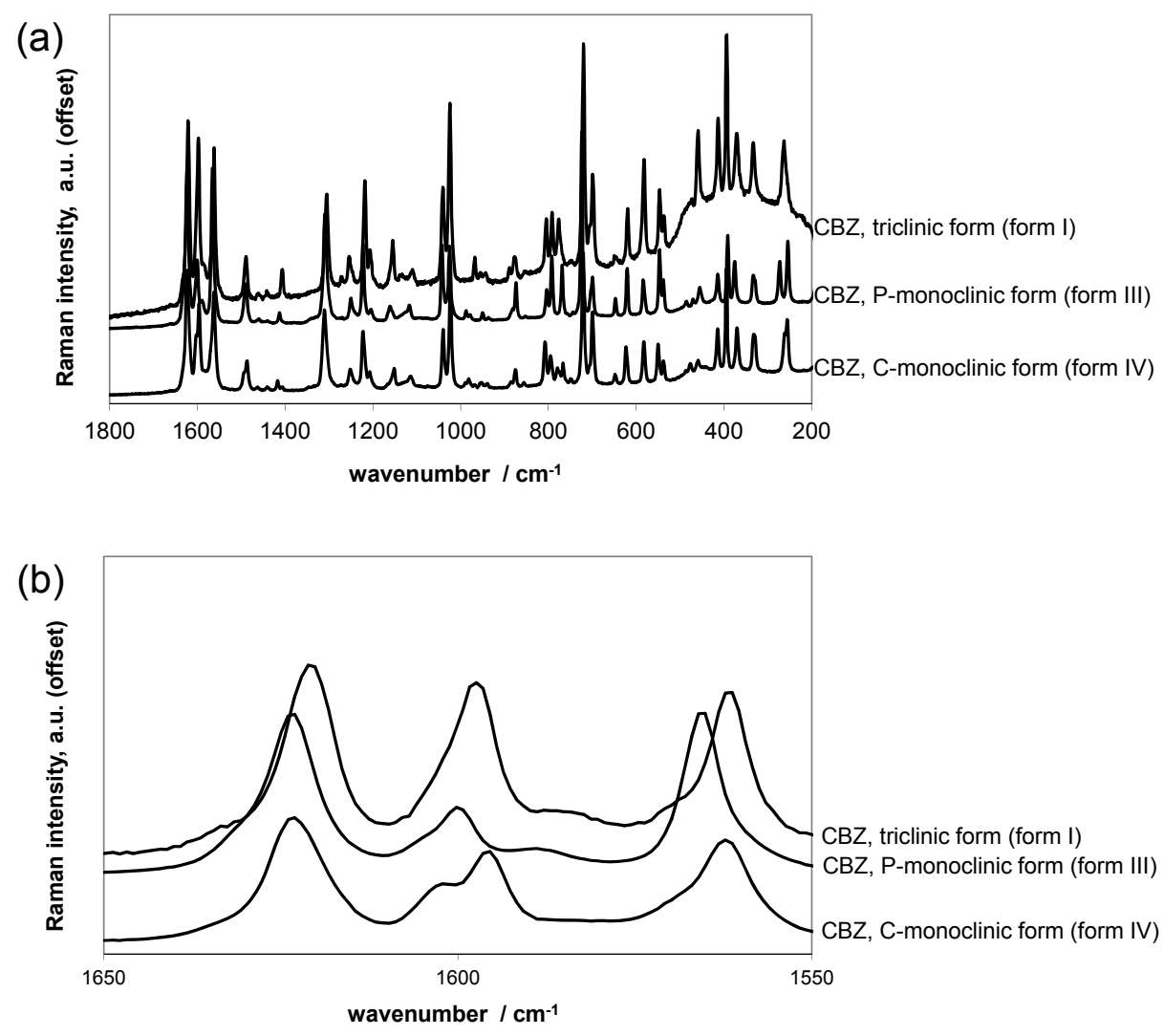

Figure 7.17. Raman spectra of CBZ Form I (top), Form III (middle) and Form IV (bottom); (a) in the whole wavenumber range and (b) wavenumber range from 1650 to $1550 \mathrm{~cm}^{-1}$. Raman intensities scaled and shifted

In some cases, such as isomorphous solvates (solvates which are identical in structure, except that different solvents occupy a certain lattice space), the specificity of IR and Raman is often superior to XRPD. 
The usefulness of Raman spectroscopy as a characterisation method for highthroughput screening (HTS) experiments is demonstrated in Figure 7.18. A cocrystal screen was performed in a 96-well plate quartz plate and the resulting solids were characterised by Raman microscopy directly in the wells. Raman microscopy allows spatial resolution in the order of 10 microns, such that spectra of individual crystals can easily be recorded. The second spectrum from the bottom is the Raman spectrum of a crystal in a particular well. It can be seen that both characteristic lines of the co-crystal former (spectrum at the bottom) and API (spectrum at the top) can be discerned in the solid which formed, indicating the presence of both molecules in the solid. On the other hand, it can be seen that some lines (highlighted by the ovals) are shifted with respect to the components (API and co-crystal former), indicating a different solid structure and thus potential co-crystal formation. This solid was therefore classified as a "co-crystal lead" and the co-crystal was reproduced on a larger scale (third spectrum from the bottom). It had the same Raman spectrum as the small crystal in the well, indicating that it was the same form, and further characterisation of this larger sample then proved that it was in fact a co-crystal.

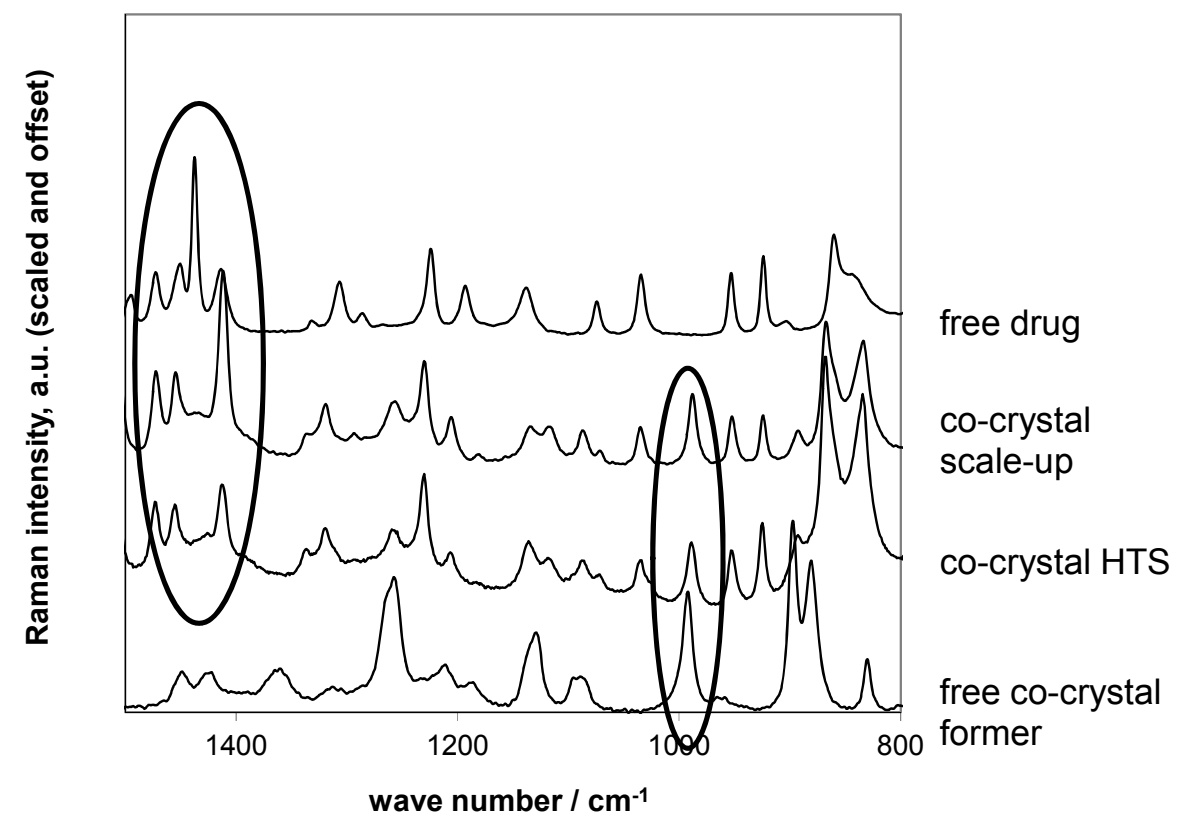

Figure 7.18. Raman spectra of the co-crystal former (L-tartaric acid), the co-crystal in a 96-well plate (recorded with a Raman microscope), the scaled-up co-crystal and the API (piracetam).

\subsection{CONCLUSION}

If an active ingredient is formulated as a solid dosage form, it is possible to choose the form of the API in the drug product from a very large number of 
possible forms. Which one of these forms is chosen may have a tremendous impact on the quality of the drug product. Bioavailability may be affected, which will have an impact on both drug efficacy and safety. Varying physical and chemical stability as well as hygroscopicity will affect the required storage conditions and maximal storage life. Non-stoichiometric hydrates may lead to problems in terms of API content, since the drug substance may contain various amounts of water, i.e. API content may not be proportional to API mass. Parameters such as bulk density, flowability, compressibility, the influence of mechanical stress on the solid form and melting point will affect the formulation process. The taste of the drug product may even be influenced (e.g. salt forms). Finally, the cost of the drug product may be influenced, since the efficiency of the crystallisation process (yield, purity, speed and removal of residual solvents) may be different for different forms.

In terms of polymorph selection, stability is generally the dominant property for the pharmaceutical industry, such that in most cases the stable polymorph will be chosen for development. Having the lowest free energy, the stable form also has the generally undesired property of being the least soluble one, but that is often considered a smaller problem than the potential conversion of a metastable form to the stable form during storage. If hydrates exist, the decision becomes more difficult, as outlined in Section 7.2.2.1.

Therefore, in order to be able to select the optimal form, a thorough characterisation is necessary, including the determination of solubility as a function of $\mathrm{pH}$ and temperature, stability, morphology, hygroscopicity, chemical purity, particle size, melting point, etc. Which of these parameters are most important will depend on the particular drug and the intended application of the drug. It makes sense to list the relevant properties for a particular drug in descending order of importance and then to compile the corresponding values of the available polymorphs, salts and co-crystals. The respective property of each form could then be graded with colours or shades of grey as "good", "intermediate" or "bad", so that a look at the corresponding matrix (Figure 7.19) would allow for choosing the optimal form.

\begin{tabular}{|l|c|c|c|c|c|c|c|}
\hline & $\begin{array}{c}\text { free drug, polym. } \\
\alpha\end{array}$ & free drug, polym. & \multicolumn{1}{|c|}{ Salt A, polym. I } & Salt B, polym. I & Co-cr X, Polym. A & Co-cr Y, Polym. A & $\ldots . .$. \\
\hline Solubility & 0.01 & 0.04 & 1 & 0.08 & 0.5 & 0.7 & \\
\hline $\begin{array}{l}\text { Thermodyn. } \\
\text { stability }\end{array}$ & yes & no & yes & yes & yes & yes & \\
\hline Hygroscopicity & $+0.1 \% @ 70 \%$ r.h. & $+0.1 \% @ 70 \%$ r.h. & $+0.1 \% @ 70 \%$ r.h. & $+2.0 \% @ 70 \%$ r.h. & channel hydrate & $+1.5 \%$ @ $70 \%$ r.h. & \\
\hline Melting Point & $80^{\circ} \mathrm{C}$ & $70^{\circ} \mathrm{C}$ & $150^{\circ} \mathrm{C}$ & $170^{\circ} \mathrm{C}$ & $150^{\circ} \mathrm{C}$ & $60^{\circ} \mathrm{C}$ & \\
\hline$\cdots .$. & & & & & & & \\
\hline
\end{tabular}

Figure 7.19. Scheme for selecting the optimal form. White indicates good, light grey intermediate and dark grey bad properties. Therefore, "Salt A, Polymorph I" would be the optimal choice here 
Naturally, in order to be able to select the optimal form, it is necessary to first produce the various forms and then to understand their properties in detail. Therefore, thorough form screening and characterisation is essential. This requires experimental effort, experience, theoretical insight and understanding. But, we certainly think that the effort invested in form screening and understanding more than pays off, since it will ensure that a better product in terms of efficacy, safety, ease of use, cost and patent protection will result in the end. 


\section{ABBREVIATIONS}

API active pharmaceutical ingredient

ATR-IR attenuated total reflectance infrared

BCS Biopharmaceutics Classification System

CBZ carbamazepine

DP drug product

DS drug substance

DSC differential scanning calorimetry

DVS dynamic vapour sorption

ET-diagram energy-temperature diagram

GRAS generally recognised as safe

HPLC high performance liquid chromatography

ICH International Conference on Harmonisation

IP intellectual property

IR infrared

NIR near infrared

NMR nuclear magnetic resonance

r.h. relative humidity

TG thermogravimetry

TG-FTIR thermogravimetry coupled to Fourier transform IR

TG-MS thermogravimetry coupled to mass spectroscopy

TMA thermomechanical analysis

TSC thermally stimulated current

XRD X-ray diffraction

XRPD X-ray powder diffraction 


\section{REFERENCES}

1. W.C. McCrone. Polymorphism. In: Physics and Chemistry of the Organic Solid State. (Eds. D. Fox, M.M. Labes and A. Weissberger) Interscience Publishers, London (1965) vol. 2, pp. 725-767.

2. International Conference on Harmonisation of Technical Requirements for Registration of Pharmaceuticals for Human Use, ICH Harmonised Tripartite Guideline. Specifications: Test Procedures and Acceptance Criteria for New Drug Substances and New Drug Product.

3. Ulrich J. Griesser, J.G. Stowell. Solid-state analysis of polymorphism. In Pharmaceutical Analysis. (Eds: D.C. Lee, M. L.Webb) Blackwell Publishing Ltd., Oxford (2003) p. 240-294.

4. P. H. Stahl, C.G. Wermuth. Handbook of Pharmaceutical Salts: Properties, Selection, and Use. Wiley-VCH, Weinheim (2002).

5. C.A. Lipinski, F. Lombarda, B.W. Dominy and P.J. Feeney. Experimental and computational approaches to estimate solubility and permeability in drug discovery and development settings. Advanced Drug Delivery Reviews 46(1) (2001) 3-26.

6. G.P. Stahly. Presentation at $22^{\text {nd }}$ SCI Process Development Symposium, Cambridge, December 2004.

7. U. J. Griesser. The importance of solvates. In "Polymorphism in the Pharmaceutical Industry" (Ed. R. Hilfiker) Wiley-VCH, Weinheim (2006) p 221-234.

8. H.G. Brittain. Polymorphism in Pharmaceutical Solids. Marcel Dekker, Inc., New York (1999).

9. S.R. Byrn, R.R Pfeiffer, J.G. Stowell. Solid State Chemistry of Drugs. $2^{\text {nd }}$ Ed., SSCI Inc., West Lafayette (1999).

10. J. Bernstein. Polymorphism in Molecular Crystals. Oxford Science Publications, Oxford (2002).

11. R. Hilfiker. Polymorphism in the Pharmaceutical Industry. Wiley-VCH, Weinheim (2006).

12. H.G. Brittain. Polymorphism in Pharmaceutical Solids. $2^{\text {nd }}$ edition, Informa Healthcare, New York (2009).

13. W.H. DeCamp. The impact of polymorphism on drug development: A regulator's viewpoint. American Pharmaceutical Review 4 (2001) 70-77.

14. B. Wunderlich and J. Grebowicz. Thermotropic mesophases and mesophase transitions of linear, flexible macromolecules. Advances in Polymer Science 60/61 (1984) 1-59.

15. B. Wunderlich. A classification of molecules, phases, and transitions as recognized by thermal analysis. Thermochimica Acta 340 (1999) 37-52.

16. E.B. Vadas, P. Toma and G. Zografi. Solid-state transitions initiated by water vapor sorption of crystalline L-660,711, a leukotriene $\mathrm{D}_{4}$ receptor antagonist. Pharmaceutical Research 8(2) (1991) 148-155.

17. K.R. Morris, A.W. Newman, D.E. Bugay, S.A. Ranadive, A.K. Singh, M. Szyper, S.A., Varia, H.G. Brittain and A.T.M. Serajuddin. Characterization of humidity-dependent changes in crystal properties of a new HMG-CoA reductase inhibitor in support of 
its dosage form development. International Journal of Pharmaceutics 108(3) (1994) 195-206.

18. C.L. Stevenson, D.B. Bennett and D. Lechuga-Ballesteros. Pharmaceutical liquid crystals: The relevance of partially ordered systems. Journal of Pharmaceutical Sciences 94(9) (2005) 1861-1880.

19. G.L. Amidon, H. Lennernas, V.P. Shah and J.R. Crison. A theoretical basis for a biopharmaceutic drug classification: The correlation of in vitro drug product dissolution and in vivo bioavailability. Pharmaceutical Research 12(3) (1995) 413420.

20. S. Lohani, D.J.W. Grant. Thermodynamics of polymorphs. In Polymorphism in the Pharmaceutical Industry (Ed. R. Hilfiker), Wiley-VCH, Weinheim (2006) p21-42.

21. K.R. Morris. Structural aspects of hydrates and solvates. In Polymorphism in Pharmaceutical Solids. (Ed. H.G. Brittain), Marcel Dekker, Inc., New York, (1999) p 125-181.

22. A.L. Grzesiak, M. Lang, K. Kim and A.J. Matzger. Comparison of the four anhydrous polymorphs of carbamazepine and the crystal structure of form I. Journal of Pharmaceutical Sciences 92(11) (2003) 2260-2271.

23. R.J. Behme and D. Brooke. Heat of fusion measurement of a low melting polymorph of carbamazepine that undergoes multiple phase changes during DSC. Journal of Pharmaceutical Sciences 80(10) (1991) 986-990.

24. T. Rager and R. Hilfiker. Stability domains of multi-component crystals in ternary phase diagrams. Zeitschrift für Physikalische Chemie 223 (2009) 793-813.

25. T. Rager and R. Hilfiker. Co-crystal formation from solvent mixtures. Crystal Growth.and Design 10(7) (2010) 3237-3241.

26. W. Ostwald. Studien über die Bildung und Umwandlung fester Körper. Zeitschrift für Physikalische Chemie 22 (1897) 289-330.

27. A. Myerson. Handbook of Industrial Crystallization. $2^{\text {nd }}$ edition. ButterworthHeinemann, Boston (2002).

28. R.J. Davey, J. Garside. From Molecules to Crystallizers. Oxford University Press, Oxford (2000).

29. N. Blagden and R. Davey. Polymorphs take shape. Chemistry in Britain 35(3) (1999) 44-47.

30. W. Beckmann, W. Otto and U. Budde. Crystallization of the stable polymorph of hydroxytriendione: Seeding process and effects of purity. Organic Process Research and Development 5(4) (2001) 387-392.

31. T. Mukuta, A.Y. Lee, T. Kawakami and A.S. Myerson. Influence of impurities on the solution-mediated phase transformation of an active pharmaceutical ingredient. Crystal Growth \& Design 5(4) (2005) 1429-1436.

32. J.K. Guillory. Generation of polymorphs, hydrates, solvates and amorphous solids. In Polymorphism in Pharmaceutical Solids. (Ed. H.G. Brittain) Marcel Dekker, New York (1999) 183-226.

33. C.-H. Gu, H. Li, R.B. Gandhi and K. Raghavan. Grouping solvents by statistical analysis of solvent property parameters: implication to polymorph screening. International Journal of Pharmaceutics 283(1) (2004) 117-125.

34. R.J. Harman. The drug development process. 1. Introduction and overview. Pharmaceutical Journal 262(7035) (1999) 334-337. 
35. M. Szelagiewicz, C. Marcolli, S. Cianferani, A.P. Hard, A. Vit, A., Burkhard, M.von Raumer, U.C. Hofmeier, A. Zilian, E. Francotte and R. Schenker. In situ characterization of polymorphic forms. Journal of Thermal Analysis and Calorimetry 57(107) (1999) 23-43.

36. W.-F. Maier, J. Klein, C. Lehmann, H.W. Schmidt. Offenlegungsschrift DE 19822077 A1, "Kombinatorisches Verfahren zur Herstellung und Charakterisierung von kristallinen und amorphen Materialbibliotheken im Mikrogramm-Massstab" (1999).

37. S. Balbach and C. Korn. Pharmaceutical evaluation of early development candidates "the $100 \mathrm{mg}$-approach". International Journal of Pharmaceutics 275(1) (2004) 1-12.

38. R. Storey, R. Docherty, P. Higginson, C. Dallman, C. Gilmore, G. Barr and W. Dong. Automation of Solid Form Screening Procedures in the Pharmaceutical Industry How to Avoid the Bottlenecks. Crystallography Reviews 10 (2004) 45-56.

39. D.L. Carlton, O.P. Dhingra, P.W. Waters. "High Throughput Crystal Form Screening Workstation and Method of Use", US patent 7,008,599 (2006).

40. S.L. Morissette, Ö. Almarsson, M.L. Peterson, J.F. Remenar, M.J. Read, A.V. Lemmo, S. Ellis, M.J. Cima and C.R. Gardner. High-throughput crystallization: polymorphs, salts, co-crystals and solvates of pharmaceutical solids. Advanced Drug Delivery Reviews 56(3) (2004) 275-300.

41. C.R. Gardner, C.T. Walsh and Ö. Almarsson. Drugs as materials: Valuing physical form in drug discovery Nature Reviews Drug Discovery 3(11) (2004) 926-934.

42. A. Van Langevelde and E. Blomsma. High-throughput screening in solid form selection. Acta Crystallographica Section A: Foundations 58 (2002) C9 (Supplement).

43. P. Desrosiers, E. Carlson, W. Chandler, H. Chau, P. Cong, R. Doolen, C. Freitag, S. Lin, C. Masui, E. Wu, T. Crevier, D. Mullins, L. Song, R. Lou, J. Zhan, A. Tangkilisan, Q. Ung and K. Phan. High throughput technologies for preformulation: Salt selection and polymorph studies. Acta Crystallographica Section A: Foundations 58 (2002) C9 (Supplement).

44. R. Hilfiker, J. Berghausen, F. Blatter, A. Burkhard, S.M. De Paul, B. Freiermuth, A. Geoffroy, U. Hofmeier, C. Marcolli, B. Siebenhaar, M. Szelagiewicz, A. Vit and M. von Raumer. Polymorphism - Integrated approach from high-throughput screening to crystallization optimization. Journal of Thermal Analysis and Calorimetry 73(2) (2003) 429-440. 